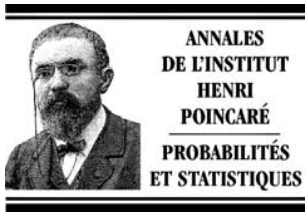

www.elsevier.com/locate/anihpb

\title{
Regularity of formation of dust in self-similar fragmentations
}

\author{
Bénédicte Haas \\ Laboratoire de probabilités et modèles aléatoires, Université Pierre et Marie Curie et CNRS UMR 7599, \\ 175, rue du Chevaleret, 75013 Paris, France
}

Received 11 June 2003; received in revised form 28 October 2003; accepted 4 November 2003

Available online 27 February 2004

\begin{abstract}
In self-similar fragmentations with a negative index, fragments split even faster as their mass is smaller, so that the fragmentation runs away and some mass is reduced to dust. Our purpose is to investigate the regularity of this formation of dust. Let $M(t)$ denote the mass of dust at time $t$. We give some sufficient and some necessary conditions for the measure $d M$ to be absolutely continuous. In case of absolute continuity, we obtain an approximation of the density by functions of small fragments. We also study the Hausdorff dimension of $d M$ and of its support, as well as the Hölder-continuity of the dust's mass $M$.
\end{abstract}

(C) 2004 Elsevier SAS. All rights reserved.

\section{Résumé}

Dans les fragmentations auto-similaires d'indice négatif, les fragments se brisent d'autant plus vite que leur masse est petite, de telle sorte que la fragmentation s'emballe et réduit de la masse à l'état de poussière. On s'intéresse ici à la régularité de la formation de la poussière. Soit $M(t)$ la masse de la poussière au temps $t$. On donne des conditions suffisantes et des conditions nécessaires pour que la mesure $d M$ soit absolument continue par rapport à la mesure de Lebesgue. Lorsque c'est le cas, on approxime la densité par des fonctions dépendant des petits fragments. On étudie également la dimension de Hausdorff de la mesure $d M$ et de son support, ainsi que la continuité Hölderienne de la masse de la poussière $M$.

(c) 2004 Elsevier SAS. All rights reserved.

MSC: 60J25; 60G17

Keywords: Fragmentation; Self-similarity; Loss of mass to dust; Lebesgue density; Hölder-continuity

\section{Introduction}

Fragmentation processes are random models for the evolution of an object that splits as time goes on. These models, together with their deterministic counterparts, have been widely studied by both mathematicians and

E-mail address: haas@ccr.jussieu.fr (B. Haas). 
physicists. We mention Aldous' survey [1] of the literature on the subject and Les Houches proceedings [10] for physical view points.

The self-similar fragmentations processes we consider in this work are those studied by Bertoin in [5-7]. Informally, a self-similar fragmentation is a process that enjoys both a fragmentation property and a scaling property. By fragmentation property, we mean that the fragments present at a time $t$ will evolve independently with break-up rates depending on their masses. The scaling property specifies these mass-dependent rates. More precisely, there is a real number $\alpha$, called index of self-similarity, such that the process starting from a fragment with mass $m$ has the same distribution as $m$ times the process starting from a fragment with mass 1, up to the time change $t \mapsto t m^{\alpha}$. The definition will be made rigorous in Section 2 .

Our interest is more specifically in self-similar fragmentations with negative indices of self-similarity, in which a loss of mass occurs (see e.g. [7]), corresponding to the appearance of dust - or microscopic fragments - whose total mass is non-zero. This phenomenon is a consequence of an intensive splitting that results from the scaling property: when $\alpha<0$, small fragments split faster than large ones, so that the average speed of splitting increases as time goes on and the fragmentation runs away and produces some dust. Let us mention [15-17,19] for discussions on the appearance of dust for some different classes of random fragmentations and for some deterministic fragmentation models.

The purpose of this paper is to study the regularity of this formation of dust. To be more precise, let $M(t)$ be the dust's mass at time $t, t \geqslant 0$. It is a non-decreasing function that can be written as $M(t)=\int_{0}^{t} d M(u)$ for some non-negative measure $d M$. Our main point of interest is to investigate the existence of a Lebesgue density for the mass measure $d M$. We are also concerned with questions such as the approximation of the density (when it exists) by functions depending on small fragments, the Hausdorff dimensions of $d M$ and $d M$ 's support when $d M$ is singular and the Hölder-continuity of the dust's mass $M$.

This study is motivated and illustrated by the "Brownian excursion fragmentation" example, introduced first in [6] and that we now roughly present. Let $e=(e(x), 0 \leqslant x \leqslant 1)$ be the normalized Brownian excursion (informally, $e$ is a Brownian motion on the unit interval, conditioned by $e(0)=e(1)=0$ and $e(x)>0$ for $0<x<1)$ and consider the family of random nested open sets of $] 0,1[$

$$
F_{e}(t)=\{x \in] 0,1[: e(x)>t\}, \quad t \geqslant 0 .
$$

This family corresponds to a fragmentation of the interval ]0, 1[ as time passes (actually, one may prove that it is a self-similar fragmentation with index $\alpha=-1 / 2$ - see [6]). The interval components of $F_{e}(t)$ are the "fragments" present at time $t$ with a positive mass (the mass of a fragment being the length of the corresponding interval) and their total mass is equal to $\int_{0}^{1} 1_{\{e(u)>t\}} d u$. The dust's mass $M_{e}(t)$ is thus equal to $\int_{0}^{1} 1_{\{e(u) \leqslant t\}} d u$, which is positive for all $t>0$. According to the Brownian motion theory, there is a local time process $\left(L_{e}(t), t \geqslant 0\right)$ such that

$$
M_{e}(t)=\int_{0}^{t} L_{e}(s) d s \quad \text { for all } t \geqslant 0, \text { a.s. }
$$

so that the mass measure $d M_{e}$ has $L_{e}$ for Lebesgue density a.s. It is further known that this density $L_{e}$ can be approximated by functions of small interval components (i.e. fragments) as follows (see e.g. [22]): for every $t \geqslant 0$,

$$
\lim _{\varepsilon \rightarrow 0} \sqrt{\frac{2 \pi}{\varepsilon}} M_{e}(t, \varepsilon) \stackrel{\text { a.s. }}{=} \lim _{\varepsilon \rightarrow 0} \sqrt{2 \pi \varepsilon} N_{e}(t, \varepsilon) \stackrel{\text { a.s. }}{=} L_{e}(t),
$$

where $M_{e}(t, \varepsilon)$ denotes the total length of excursions intervals of $e$ above $t$ of length less or equal to $\varepsilon$ (that is, in terms of fragments, the total mass of fragments present at time $t$ having a mass in $] 0, \varepsilon]$ ); and $N_{e}(t, \varepsilon)$ is the number of excursions of $e$ above $t$ of length greater then $\varepsilon$ (i.e. the number of fragments present at time $t$ of mass greater than $\varepsilon$ ). Another point we are interested in, as mentioned above, is the Hölder-continuity of the dust's mass $M_{e}$. It is well-known that the local time $L_{e}$ is bounded a.s.: the dust's mass $M_{e}$ is therefore Lipschitz a.s. 
Miermont [21] constructs similarly some fragmentations from the normalized excursions of some random continuous processes possessing a local time, which gives some more examples of fragmentations with absolutely continuous mass measure $d M$.

Our goal is to see how these regularity results extend to general self-similar fragmentations with negative indices. The paper is organized as follows. In Section 2, self-similar fragmentations are introduced and their main properties recalled. Section 3 concerns some preliminary results on the dust's mass $M$ and on tagged fragments, a tagged fragment being a fragment containing a point tagged at random, independently of the fragmentation. The evolution of such fragments is well-known and is closely connected to the mass $M$ as we shall see later. Following one or several tagged fragments as time passes will then be a key tool in the study of regularity.

There are some self-similar fragmentations for which the mass measure $d M$ does not have a Lebesgue density. Section 4 presents some sufficient (respectively necessary) conditions for $d M$ to be absolutely continuous. These conditions are stated in terms of the index of self-similarity $\alpha$ and of a dislocation measure, introduced in Section 2, that, roughly, describes the distribution of sudden dislocations. For a large class of fragmentations the critical value is $\alpha=-1$, in the sense that almost surely $d M$ has a Lebesgue density if and only if $\alpha>-1$. The sufficient conditions' proofs are coarser than the necessary ones and rely on Fourier analysis.

For fragmentations with an absolutely continuous mass measure $d M$, the approximation of the density is discussed in Section 5. Let $L(t):=d M(t) / d t$. In most cases, we prove the existence of a finite deterministic constant $C$ such that for a.e. $t$, the functions $\varepsilon^{\alpha} M(t, \varepsilon)$ and $\varepsilon^{1+\alpha} N(t, \varepsilon)$ converge a.s. to $C L(t)$ as $\varepsilon \rightarrow 0$. As in the Brownian excursion fragmentation, $M(t, \varepsilon)$ denotes the total mass of fragments of mass in $] 0, \varepsilon]$ at time $t$ and $N(t, \varepsilon)$ the number of fragments of mass greater than $\varepsilon$ at time $t$.

Section 6 is devoted to the Hölder-continuity of the dust's mass $M$ and, in cases where $d M$ is singular, to its Hausdorff dimension and that of its support. The paper ends with Appendix A containing a technical proof of a result stated in Section 3.

\section{Background on self-similar fragmentations}

Since for us the only distinguishing feature of a fragment is its mass, the fragmentation system is characterized at a given time $t$ by the ranked sequence $s_{1} \geqslant s_{2} \geqslant \cdots \geqslant 0$ of masses of fragments present at that time. Starting from a single object with mass one, the appropriate space for our models is then $\mathcal{S}^{\downarrow}$, the state of non-increasing non-negative sequences with total sum at most 1, i.e.

$$
\mathcal{S}^{\downarrow}:=\left\{s=\left(s_{i}\right)_{i \in \mathbb{N}^{*}}, s_{1} \geqslant s_{2} \geqslant \cdots \geqslant 0: \sum_{i=1}^{\infty} s_{i} \leqslant 1\right\},
$$

endowed with the topology of pointwise convergence. The difference $1-\sum_{i} s_{i}$ may be thought as the mass of dust.

Definition. Let $(X(t), t \geqslant 0)$ be a $\mathcal{S}^{\downarrow}$-valued Markov process continuous in probability and denote by $P_{r}$, $0<r \leqslant 1$, the law of $X$ starting from $(r, 0, \ldots)$.

(i) The process $X$ is a fragmentation process if for each $t_{0} \geqslant 0$, conditionally on $X\left(t_{0}\right)=\left(s_{1}, s_{2}, \ldots\right)$, the process $\left(X\left(t+t_{0}\right), t \geqslant 0\right)$ has the same law as the process obtained, for each $t \geqslant 0$, by ranking in the decreasing order the components of sequences $X^{1}(t), X^{2}(t), \ldots$, where the r.v. $X^{i}$ are independent with respective laws $P_{s_{i}}$.

(ii) If further $X$ enjoys the scaling property, which means that there exists a real number $\alpha$, called index of self-similarity, such that the law of $(X(t), t \geqslant 0)$ under $P_{r}$ is the same as that of $\left(r X\left(t r^{\alpha}\right), t \geqslant 0\right)$ under $P_{1}$, then $X$ is a self-similar fragmentation process with index $\alpha$. When $\alpha=0, X$ is called a homogeneous fragmentation process. 
We consider fragmentation processes starting from $X(0)=(1,0,0, \ldots)$ and denote by $X_{i}(t), i \geqslant 1$, the components of the sequence $X(t), t \geqslant 0$. Let $\mathcal{F}=(\mathcal{F}(t), t \geqslant 0)$ be the natural filtration generated by $X$, completed up to $P$-null sets. According to [3], the fragmentation property holds moreover for $\mathcal{F}$-stopping times and we shall refer to it as the strong fragmentation property.

In [6], Bertoin shows that each self-similar fragmentation possesses a càdlàg version - which we may always consider - and that its distribution is entirely characterized by three parameters: the index of self-similarity $\alpha$, an erosion coefficient $c \geqslant 0$ and a dislocation measure $v$, which is a sigma-finite measure on $\mathcal{S} \downarrow$ that does not charge $(1,0, \ldots)$ and such that

$$
\int_{\mathcal{S} \downarrow}\left(1-s_{1}\right) v(d s)<\infty .
$$

Roughly speaking, the erosion is a deterministic continuous phenomenon and the dislocation measure describes the rates of sudden dislocations: a fragment with mass $x$ splits in fragments with mass $x s, s \in \mathcal{S}^{\downarrow}$, at rate $x^{\alpha} v(d s)$. Conversely, given $\alpha, c, v$ satisfying the requirements above, one can construct a corresponding selfsimilar fragmentation.

For technical reasons, we may need to work with an interval representation of the fragmentation: by combination of results of [3] and [6], every $\alpha$-self-similar fragmentation $X$ can be constructed from a family $(F(t), t \geqslant 0)$ of nested random open sets of ]0,1[ so that, for every $t \geqslant 0, X(t)=\left(X_{1}(t), \ldots\right)$ is the ordered sequence of the lengths of the interval components of $F(t)$. This process $F$ possesses both the $\alpha$-self-similarity and fragmentation properties (we refer to [6] for precise definitions) and is called an interval representation of $X$. There is actually a one-to-one correspondence between the laws of $\mathcal{S}^{\downarrow}$-valued and interval-valued self-similar fragmentations.

The advantage of this interval's view point is the passage from homogeneous to self-similar fragmentations by appropriate time-changes: consider a homogeneous interval fragmentation $\left(F^{0}(t), t \geqslant 0\right)$ and define by $I_{x}(t)$ the interval component of $F^{0}(t)$ that contains $x$ if $x \in F^{0}(t)$ and set $I_{x}(t):=\emptyset$ if $x \notin F^{0}(t), x$ in ]0, 1[. Then introduce the time-changed functions

$$
T_{x}^{\alpha}(t):=\inf \left\{u \geqslant 0: \int_{0}^{u}\left|I_{x}(r)\right|^{-\alpha} d r>t\right\},
$$

and consider the family of nested open sets of $] 0,1[$ defined by

$$
F^{\alpha}(t)=\bigcup_{x \in] 0,1[} I_{x}\left(T_{x}^{\alpha}(t)\right), \quad t \geqslant 0
$$

As proved in [6], $F^{\alpha}$ is an $\alpha$-self-similar interval fragmentation and each self-similar interval fragmentation can be constructed like this from a homogeneous one. This associated homogeneous fragmentation has the same dislocation measure and erosion coefficient as the self-similar fragmentation.

This interval setting is particularly appropriate to tag fragments at random as explained in detail in the following section.

\section{Tagged fragments and dust's mass}

From now on, we shall focus on self-similar fragmentations such that

$$
\alpha<0, \quad c=0, \quad v \neq 0 \quad \text { and } \quad \nu\left(\sum_{i} s_{i}<1\right)=0 .
$$


That $v\left(\sum_{i} s_{i}<1\right)=0$ means that no mass is lost within sudden dislocations and $c=0$ means there is no erosion. In terms of the fragmentation $X$, the dust's mass at time $t$ then writes

$$
M(t)=1-\sum_{i=1}^{\infty} X_{i}(t) .
$$

The index $\alpha$ being negative, we know by Proposition 2 in [7], that with probability one $M$ is càdlàg, non-decreasing and reaches 1 in finite time. It can then be viewed as the distribution function of some random probability measure, that we denote by $d M$ :

$$
M(t)=\int_{0}^{t} d M(u), \quad t \geqslant 0 .
$$

A useful tool to study this mass of dust is to tag a fragment at random in the fragmentation. To do so, consider $F$ an interval representation of $X$ as recalled in the previous section andlet $U$ be a random variable uniformly distributed on ]0, 1[ and independent of $F$. At each time $t$, if $U \in F(t)$, denote by $\lambda(t)$ the length of the interval component of $F(t)$ containing $U$. If $U \notin F(t)$, set $\lambda(t):=0$. Bertoin, in [5] and [6], has determined the law of the process $\lambda$ :

$$
\lambda \stackrel{\text { law }}{=} \exp \left(-\xi_{\tau(.)}\right)
$$

where $\xi$ is a subordinator with Laplace exponent $\phi$ given for all $q \geqslant 0$ by

$$
\phi(q)=\int_{\mathcal{S} \downarrow}\left(1-\sum_{i=1}^{\infty} s_{i}^{1+q}\right) v(d s),
$$

and $\tau$ is the time-change

$$
\tau(t)=\inf \left\{u \geqslant 0: \int_{0}^{u} \exp \left(\alpha \xi_{r}\right) d r>t\right\}, \quad t \geqslant 0 .
$$

We refer to [4] for background on subordinators and recall that $E\left[\mathrm{e}^{-q \xi_{r}}\right]=\mathrm{e}^{-r \phi(q)}$ for $r, q \geqslant 0$. Remark that formula (4) defines in fact a function $\phi$ on $\mathbb{R}$ such that $\phi(q) \in[0, \infty[$ for $q \geqslant 0$ and $\phi(q) \in[-\infty, 0[$ for $q<0$. Let $\rho$ be the largest $q$ such that $\phi(-q)>-\infty$. Since $v$ integrates $\left(1-s_{1}\right)$, this definition is equivalent to

$$
\rho=\sup \left\{q \geqslant 0: \int_{\mathcal{S} \downarrow} \sum_{i=2}^{\infty} s_{i}^{1-q} v(d s)<\infty\right\} .
$$

Here we use the convention $0^{-a}=\infty$ for $a>0$. Hence, when $q>1$ the series $\sum_{i=2}^{\infty} s_{i}^{1-q}=\infty$ for any sequence in $\mathcal{S}^{\downarrow}$ and consequently $\rho \leqslant 1$. The Hölder-continuity of the dust's mass $M$, studied in Section 6.2, depends on this coefficient $\rho$.

The law of the first time $I$ at which the tagged fragment is reduced to dust, i.e.

$$
I:=\inf \{t \geqslant 0: \lambda(t)=0\},
$$

can then be expressed as a function of $\alpha$ and $\xi$ :

$$
I \stackrel{\text { law }}{=} \int_{0}^{\infty} \exp \left(\alpha \xi_{r}\right) d r
$$


One first important example of the use of tagged fragments is that the dust's mass $M$ then coincides with the distribution function of $I$ conditional on $X$, that is, a.s.

$$
M(t)=P(I \leqslant t \mid X), \quad t \geqslant 0 .
$$

Indeed, $I \leqslant t$ if and only if $U \notin F(t)$ and the conditional probability of this event given $X$ is the total length of ] $0,1\left[\backslash F(t)\right.$, i.e. $1-X_{1}(t)-X_{2}(t)-\cdots=M(t)$. The point is that the law of $I$ has been extensively studied (see e.g. $[13,9])$ and it will therefore give us some information on $M$.

The rest of the section concerns some preliminary results that will be needed in the sequel. Section 3.1 deals with some regularity properties of I's distribution. The main results of Carmona et al. [13] are recalled and some other properties developed. In Section 3.2, we tag several fragments independently and study their masses at the first time at which some tagged fragments are different. Section 3.3 is devoted to the first time at which all the mass is reduced to dust.

\subsection{On the regularity of I's distribution}

By (6), $I$ has the same law as $\int_{0}^{\infty} \exp \left(\alpha \xi_{r}\right) d r$. Carmona, Petit and Yor studied in [13] these exponential functionals. They showed (Proposition 3.1 iv, Proposition 3.3) that $I$ has entire moments of all positive orders and that

$$
\mu:=E\left[\xi_{1}\right]=\frac{1}{|\alpha|} E\left[I^{-1}\right] .
$$

Remark with (4), that

$$
\mu=E\left[\xi_{1}\right]=\phi^{\prime}\left(0^{+}\right)=\int_{\mathcal{S} \downarrow}\left(\sum_{i=1}^{\infty}\left|\log \left(s_{i}\right)\right| s_{i}\right) v(d s) .
$$

In the sequel, we will often assume that $\mu<\infty$, because of the following lemma:

Lemma 1. Suppose that $\mu<\infty$ and $\int_{\mathcal{S} \downarrow}\left(1-s_{1}\right)^{\beta} v(d s)<\infty$ for some $\beta<1$. Then, there is an infinitely differentiable function $k:] 0, \infty[\rightarrow[0, \infty[$ such that

(i) $P(I \in d x)=k(x) d x$,

(ii) for all $a \geqslant 0$, the function $x \mapsto x^{a} k(x)$ is bounded on $] 0, \infty[$.

We point out that the existence of some $\beta<1$ such that $\int_{\mathcal{S} \downarrow}\left(1-s_{1}\right)^{\beta} \nu(d s)<\infty$ is not necessary to prove the assertion (i).

Proof. (i) It is Proposition 2.1 of [13].

(ii) The point is to show that for all $a \geqslant 0$, the function $x \mapsto \mathrm{e}^{a x} k\left(\mathrm{e}^{x}\right)$ is bounded on $\mathbb{R}$. To that end, we need the following result of [13] (Proposition 2.1): the density $k$ is a solution of the equation

$$
k(x)=\int_{x}^{\infty} \bar{\pi}\left(\frac{1}{|\alpha|} \log \left(\frac{u}{x}\right)\right) k(u) d u, \quad x>0,
$$

where $\pi$ denotes the Lévy measure of $\xi$ and $\bar{\pi}(x):=\pi(] x, \infty[), x>0$. This leads to

$$
\begin{aligned}
\mathrm{e}^{a x} k\left(\mathrm{e}^{x}\right) & =\int_{-\infty}^{\infty} 1_{\{u-x>0\}} \bar{\pi}((u-x) /|\alpha|) \mathrm{e}^{a(x-u)} \mathrm{e}^{(a+1) u} k\left(\mathrm{e}^{u}\right) d u \\
& =\left(1_{\{\cdot<0\}} \bar{\pi}(-\cdot /|\alpha|) \mathrm{e}^{a \cdot} * \mathrm{e}^{(a+1) \cdot} k\left(\mathrm{e}^{\cdot}\right)\right)(x),
\end{aligned}
$$


where $*$ denotes the convolution product. It is well-known (by Hölder inequality) that for $p \geqslant 1$ the convolution product of a function of $L^{p}(d x)$ with a function of $L^{p /(p-1)}(d x)$ is bounded on $\mathbb{R}$. So if we prove that the functions $x \mapsto 1_{\{x<0\}} \bar{\pi}(-x /|\alpha|) \mathrm{e}^{a x}$ and $x \mapsto \mathrm{e}^{(a+1) x} k\left(\mathrm{e}^{x}\right)$ respectively belong to $L^{p}(d x)$ and $L^{p /(p-1)}(d x)$ for some $p \geqslant 1$, the proof will be ended.

Let us first show that $\bar{\pi} \in L^{\gamma}(d x)$ for all $1<\gamma<1 / \beta$ such that $\int_{\mathcal{S} \downarrow}\left(1-s_{1}\right)^{\beta} \nu(d s)<\infty$ (such $\beta$ exists by assumption). To see this, note that

$$
\left.\pi(d x)=\mathrm{e}^{-x} v\left(-\log \left(s_{1}\right) \in d x\right) \quad \text { on }\right] 0, \log 2[
$$

(see e.g. the remarks at the end of [5]), which gives

$$
\int_{0}^{\log 2} x^{c} \pi(d x)=\int_{\mathcal{S} \downarrow} 1_{\left\{s_{1}>1 / 2\right\}} s_{1}\left|\log s_{1}\right|^{c} v(d s), \quad c \in \mathbb{R} .
$$

Then combine this with $\int_{0}^{\infty} x \pi(d x)=\phi^{\prime}\left(0^{+}\right)<\infty$ (which is a consequence of $\mu<\infty$ and (4)) to get that $\int_{0}^{\infty}\left(x^{\beta} \vee x\right) \pi(d x)<\infty$ for the $\beta<1$ such that $\int_{\mathcal{S} \downarrow}\left(1-s_{1}\right)^{\beta} \nu(d s)<\infty$. Therefore, there exists $C>0$ such that $\bar{\pi}(x) \leqslant C\left(x^{-1} \wedge x^{-\beta}\right)$ for $x>0$. Then $\bar{\pi}$, and a fortiori $x \mapsto 1_{\{x<0\}} \bar{\pi}(-x /|\alpha|) \mathrm{e}^{a x}$, belongs to $L^{\gamma}(d x)$ for all $1<\gamma<1 / \beta$.

It remains to prove that for all $a \geqslant 0$, the function $x \mapsto \mathrm{e}^{(a+1) x} k\left(\mathrm{e}^{x}\right)$ belongs to $L^{\gamma /(\gamma-1)}(d x)$ for some $\gamma \in] 1,1 / \beta$ [. Fix such a $\gamma$ and remark that it is sufficient to show that this function belongs to $L^{\gamma^{n}}(d x)$ for all $n \in \mathbb{N}$ (because $L^{1} \cap L^{\gamma^{n}} \subset L^{\gamma /(\gamma-1)}$ when $\left.\gamma^{n} \geqslant \gamma /(\gamma-1) \geqslant 1\right)$. We prove this by induction on $n$. For $n=0$, this is an immediate consequence of $\int_{-\infty}^{\infty} \mathrm{e}^{(a+1) u} k\left(\mathrm{e}^{u}\right) d u=E\left[I^{a}\right]$, which is finite for all $a \geqslant 0$ by Proposition 3.3 of [13]. For the next step, we need the following result: for all $p, q \geqslant 1$,

$$
\text { if } f \in L^{p}(d x) \cap L^{1}(d x) \text { and if } g \in L^{q}(d x), \quad \text { then } f * g \in L^{p q}(d x),
$$

which we first prove. By applying Hölder inequality twice, first to the measure $|f(x-y)| d y$ and second to $|g(y)|^{q} d y$, we get

$$
\begin{aligned}
|f * g(x)| & \leqslant\left(\int_{-\infty}^{\infty}|g(y)|^{q}|f(x-y)| d y\right)^{1 / q}\left(\int_{-\infty}^{\infty}|f(x-y)| d y\right)^{(q-1) / q} \\
& \leqslant\left(\int_{-\infty}^{\infty}|g(y)|^{q}|f(x-y)|^{p} d y\right)^{1 / p q}\left(\int_{-\infty}^{\infty}|g(y)|^{q} d y\right)^{(p-1) / p q}\left(\int_{-\infty}^{\infty}|f(x-y)| d y\right)^{(q-1) / q} .
\end{aligned}
$$

The last two integrals do not depend on $x$ and are finite. The first integral, seen as a function of $x$, is integrable by Fubini's Theorem. So, $f * g \in L^{p q}(d x)$. Now we apply this result to functions $x \mapsto 1_{\{x<0\}} \bar{\pi}(-x /|\alpha|) \mathrm{e}^{a x}$ and $x \mapsto \mathrm{e}^{(a+1) x} k\left(\mathrm{e}^{x}\right)$, which belong respectively to $L^{\gamma}(d x)$ and $L^{1}(d x)$, and this shows with (9) that $x \mapsto \mathrm{e}^{a x} k\left(\mathrm{e}^{x}\right) \in$ $L^{\gamma}(d x)$ for $a \geqslant 0$. Applying this recursively, we get that the function $x \mapsto \mathrm{e}^{a x} k\left(\mathrm{e}^{x}\right) \in L^{\gamma^{n}}(d x)$ for all $a \geqslant 0$ and $n \in \mathbb{N}$.

\subsection{Tagging $n$ fragments independently}

We consider the joint behavior of $n$ fragments tagged independently. More precisely, let $U_{1}, \ldots, U_{n}$ be $n$ independent random variables, uniformly distributed on $] 0,1[$ and independent of the fragmentation process, and for $i=1, \ldots, n$ and $t \geqslant 0$, let $\lambda_{i}(t)$ be the length of the interval component of $F(t)$ containing the point $U_{i}$ if $U_{i} \in F(t)$ and set $\lambda_{i}(t):=0$ if $U_{i} \notin F(t)$. The law of $\left(\lambda_{1}, \lambda_{2}, \ldots, \lambda_{n}\right)$ is exchangeable, but the processes $\lambda_{1}, \lambda_{2}, \ldots, \lambda_{n}$ are not independent. They coincide on $\left[0, T_{n}\right.$ [, where $T_{n}$ denotes the first time at which the $U_{i}$ 's, $i=1, \ldots, n$, do not all belong to the same fragment, that is

$$
T_{n}:=\sup \left\{t \geqslant 0: U_{1}, \ldots, U_{n} \in \text { same interval component of } F(t)\right\} .
$$


Note that $T_{n}>0$ a.s., since, by independence of the $U_{i}$ 's, $P\left(T_{n}>t \mid \lambda_{1}\right) \stackrel{\text { a.s. }}{=} \lambda_{1}(t)^{n-1}$ which tends to 1 as $t \rightarrow 0$. At time $T_{n}$, there are $L$ distinct tagged fragments - for some random $L \geqslant 2$ - which, according to the fragmentation and scaling properties, evolve independently and with a law depending on their masses. The aim of this subsection is to give some information on these masses.

Consider an integer $l \geqslant 2$. Conditionally on $L=l$, we may assume, by exchangeability, that $U_{1}, U_{2}, \ldots, U_{l}$ belong all to different fragments at time $T_{n}$, so that the masses of the $l$ distinct tagged fragments at time $T_{n}$ are $\lambda_{1}\left(T_{n}\right), \lambda_{2}\left(T_{n}\right), \ldots, \lambda_{l}\left(T_{n}\right)$. For each $l$-tuple $\left(n_{1}, n_{2}, \ldots, n_{l}\right) \in(\mathbb{N} \backslash\{0\})^{l}$ such that $n_{1}+n_{2}+\cdots+n_{l}=n$, define then by $A_{\left(n_{1}, \ldots, n_{l}\right)}$ the event

$$
A_{\left(n_{1}, \ldots, n_{l}\right)}:=\left\{\begin{array}{l}
L=l \text { and at time } T_{n}, \text { there are } n_{k} \text { tagged points } \\
\text { in the fragment containing } U_{k}, 1 \leqslant k \leqslant l
\end{array}\right\} .
$$

The following lemma provides an integrability property of a function depending on the masses of tagged fragments at time $T_{n}$. It will be a key point in the study of regularity. More precisely, it will be used to prove the Höldercontinuity of the dust's mass $M$ (see Section 6) and, in the special case where $n=2$, to show the absolute continuity of the mass measure $d M$ for some $(\alpha, v)$-fragmentations (see Section 4).

Lemma 2. For all $a_{1}, \ldots, a_{l}$ in $\mathbb{R}$, the following assertions are equivalent:

(i) $E\left[\prod_{k=1}^{l} \lambda_{k}^{-a_{k}}\left(T_{n}\right) 1_{\left\{\lambda_{1}\left(T_{n}\right) \geqslant \lambda_{2}\left(T_{n}\right) \geqslant \cdots \geqslant \lambda_{l}\left(T_{n}\right)\right\}} 1_{\left\{A_{\left(n_{1}, n_{2}, \ldots, n_{l}\right)}\right\}}\right]<\infty$,

(ii) $\sum_{k=1}^{l} a_{k}<n-1$ and $\int_{\mathcal{S} \downarrow} \sum_{i_{1}<i_{2}<\cdots<i_{l}} \prod_{k=1}^{l} s_{i_{k}}^{n_{k}-a_{k}} 1_{\left\{s_{i_{k}}>0\right\}} v(d s)<\infty$.

The proof of this technical result is provided in Appendix A at the end of the paper.

\subsection{First time at which all the mass is reduced to dust}

The first time at which the mass is entirely reduced to dust, i.e.

$$
\mathcal{T}:=\inf \left\{t \geqslant 0: X_{1}(t)=0\right\}
$$

is almost surely finite (see [7]). The asymptotic behavior of $P(\mathcal{T}>t)$ as $t \rightarrow \infty$ is discussed in [17] and leads us to

Lemma 3. $E[\mathcal{T}]<\infty$ and $P(\mathcal{T}>t)<1$ for every $t>0$.

Proof. According to Section 5.3 in [17], there exist two positive finite constants $A$ and $B$ such that

$$
P(\mathcal{T}>t) \leqslant A \mathrm{e}^{-B t}, \quad \text { for all } t \geqslant 0 .
$$

That $E[\mathcal{T}]<\infty$ is then immediate. To prove the second assertion, assume first that

$$
\{t>0: P(\mathcal{T}<t)=0\} \neq \emptyset
$$

and denote by $t_{0}$ its largest element. Define then $u$ by $\left(t_{0}-u\right) / t_{0}=1 / 2^{|\alpha|}$. Since $u<t_{0}, \mathcal{T} \geqslant u$ a.s. Thus, applying the fragmentation and scaling properties at time $u$,

$$
\mathcal{T}=u+\sup _{1 \leqslant i<\infty} X_{i}^{|\alpha|}(u) \mathcal{T}^{(i)}
$$

where the $\mathcal{T}^{(i)}$ are iid with the same law as $\mathcal{T}$ and independent of $\mathcal{F}(u)$. In other words, if (12) holds, then for all $\varepsilon \in] 0, t_{0}-u[$,

$$
\prod_{i} P\left(X_{i}^{|\alpha|}(u) \mathcal{T}^{(i)} \leqslant t_{0}-u-\varepsilon \mid \mathcal{F}(u)\right)=P\left(\mathcal{T} \leqslant t_{0}-\varepsilon \mid \mathcal{F}(u)\right) \stackrel{\text { a.s. }}{=} 0 .
$$


To prove the statement, we therefore have to show that (13) is false. In that aim, suppose first that

$$
\left.P\left(X_{1}^{|\alpha|}(u) \mathcal{T}^{(1)} \leqslant t_{0}-u-\varepsilon \mid \mathcal{F}(u)\right) \stackrel{\text { a.s. }}{=} 0 \quad \text { for all } \varepsilon \in\right] 0, t_{0}-u[.
$$

By definition of $t_{0}$ and $u$, this implies that a.s. $\left(t_{0}-u\right) / X_{1}^{|\alpha|}(u) \leqslant t_{0}$ and then $X_{1}(u) \geqslant 1 / 2$. Using the connections between homogeneous fragmentations and self-similar ones as explained in Section 2, we see that this leads to the existence of a homogeneous fragmentation $X^{h}$ with dislocation measure $v$ such that a.s. for all $t \geqslant 0$, $X_{1}^{h}(t) \geqslant X_{1}(t)$. In particular, $X_{1}^{h}(u) \geqslant 1 / 2$ a.s. From Proposition 12 in [3] and its proof, we know the existence of a subordinator $\sigma$ with Laplace exponent given by (4) such that $X_{1}^{h}=\exp (-\sigma)$ on $[0, u]$. We then have $\sigma(u) \leqslant \ln 2$ a.s. However, it is well known that the jump process of $\sigma$ is a Poisson point process with intensity the Lévy measure of $\sigma$ and since here this Lévy measure is not trivial and $u>0$, the r.v. $\sigma(u)$ can not have a deterministic upper bound. Thus (14) can not be true and for some $\varepsilon_{0}$ in $] 0, t_{0}-u\left[, P\left(X_{1}^{|\alpha|}(u) \mathcal{T}^{(1)} \leqslant t_{0}-u-\varepsilon_{0} \mid \mathcal{F}(u)\right)>0\right.$ with a positive probability. Since $P\left(X_{i}^{|\alpha|}(u) \mathcal{T}^{(i)} \leqslant t_{0}-u-\varepsilon_{0} \mid \mathcal{F}(u)\right) \nearrow 1$ as $i \nearrow \infty$, this would imply, if (13) holds, that the sum

$$
\sum_{i}\left(1-P\left(X_{i}^{|\alpha|}(u) \mathcal{T}^{(i)} \leqslant t_{0}-u-\varepsilon_{0} \mid \mathcal{F}(u)\right)\right)
$$

diverges on the event $\left\{P\left(X_{1}^{|\alpha|}(u) \mathcal{T}^{(1)} \leqslant t_{0}-u-\varepsilon_{0} \mid \mathcal{F}(u)\right)>0\right\}$, which has positive probability. Yet, this is not possible: by (11),

$$
\begin{aligned}
\sum_{i} P\left(X_{i}^{|\alpha|}(u) \mathcal{T}^{(i)}>t_{0}-u-\varepsilon_{0} \mid \mathcal{F}(u)\right) & \leqslant A \sum_{i} \mathrm{e}^{-B\left(t_{0}-u-\varepsilon_{0}\right) X_{i}^{\alpha}(u)} 1_{\left\{X_{i}(u)>0\right\}} \\
& \leqslant A C \sum_{i} X_{i}(u) \text { a.s. }
\end{aligned}
$$

where $C:=\sup _{0 \leqslant x<\infty} x^{-1} \mathrm{e}^{-B\left(t_{0}-u-\varepsilon_{0}\right) x^{\alpha}}<\infty$. Since $\sum_{i} X_{i}(t) \leqslant 1$ a.s., sum $(15)$ is then finite a.s. and consequently (13) is false.

\section{Regularity of the mass measure $d M$}

This section is devoted to the study of existence or absence of a Lebesgue density for the mass measure $d M$ of a fragmentation $X$ with parameters $\alpha, c$ and $v$ satisfying hypothesis $(\mathrm{H})$. More precisely, we give some sufficient conditions on $\alpha$ and $v$ for the existence of a density in $L^{2}(d t \otimes d P)$ and some sufficient conditions for the measure $d M$ to be singular a.s. In the sequel, we will often assume ${ }^{1}$ that the constant $\mu$ introduced in (8) is finite, i.e.

$$
\mu=\int_{\mathcal{S} \downarrow}\left(\sum_{i=1}^{\infty}\left|\log \left(s_{i}\right)\right| s_{i}\right) v(d s)=\frac{1}{|\alpha|} E\left[I^{-1}\right]<\infty
$$

and that

$$
\int_{\mathcal{S} \downarrow}\left(1-s_{1}\right)^{\beta} \nu(d s)<\infty \quad \text { for some } \beta<1 .
$$

We recall that $I$ is a random variable that corresponds to the first time at which a tagged fragment vanishes and that its distribution is given by (6). Here is our main result:

\footnotetext{
${ }^{1}$ These assumptions (A1) and (A2) hold as soon as $\rho>0$ ( $\rho$ defined by (5)). However, it is easy to find some fragmentations for which $\rho=0$ and (A1) and (A2) hold nonetheless.
} 
Theorem 4. Suppose (A1).

(i) If (A2) holds, $\alpha>-1$ and $\int_{\mathcal{S} \downarrow} \sum_{i<j} s_{i}^{1+\alpha} s_{j} v(d s)<\infty$, then the measure $d M$ is absolutely continuous a.s. and its density belongs to $L^{2}(d t \otimes d P)$.

(ii) If $\alpha \leqslant-1$, then $d M$ is singular a.s.

In (i), the criterion $\int_{\mathcal{S} \downarrow} \sum_{i<j} s_{i}^{1+\alpha} s_{j} v(d s)<\infty$ is optimal in the sense that there are some fragmentations satisfying assumptions (A1) and (A2) on $v$, with index $\alpha>-1$ and $\int_{\mathcal{S} \downarrow} \sum_{i<j} s_{i}^{1+\alpha} s_{j} v(d s)=\infty$, and such that $d M$ is not absolutely continuous with a density in $L^{2}(d t \otimes d P)$. Some illustrating examples are given after the proof of Theorem 4(i).

In the special case where $v\left(s_{N+1}>0\right)=0$ for some given $N \geqslant 2$ (that is each dislocation gives rise to at most $N$ fragments), note that when $\alpha>-1$,

$$
\int_{\mathcal{S} \downarrow} \sum_{i<j} s_{i}^{1+\alpha} s_{j} v(d s) \leqslant \int_{\mathcal{S} \downarrow}(N-1) \sum_{2 \leqslant j \leqslant N} s_{j} v(d s) \leqslant(N-1) \int_{\mathcal{S} \downarrow}\left(1-s_{1}\right) v(d s)<\infty .
$$

Both parts of Theorem 4 then complement each other and give the following result.

Corollary 5. Assume that $v\left(s_{N+1}>0\right)=0$ for some integer $N$ and that (A1) and (A2) hold. Then, with probability one, the measure $d M$ is absolutely continuous if and only if $\alpha>-1$. When $\alpha>-1$, the density of $d M$ is in $L^{2}(d t \otimes d P)$ and when $\alpha \leqslant-1, d M$ is singular a.s.

We now turn to the proofs. That of Theorem 4(i) uses Fourier analysis.

Proof of Theorem 4(i). Introduce the Fourier transform of $d M$, i.e.

$$
\widehat{M}(\theta)=\int_{0}^{\infty} \mathrm{e}^{i \theta t} d M(t), \quad \theta \in \mathbb{R} .
$$

It is well-known that the measure $d M$ is absolutely continuous with a density $L$ in $L^{2}(d t)$ if and only if the integral $\int_{-\infty}^{\infty}|\widehat{M}(\theta)|^{2} d \theta$ is finite and then that $\int_{-\infty}^{\infty}|\widehat{M}(\theta)|^{2} d \theta=\int_{0}^{\infty} L^{2}(t) d t$. Consequently, taking the expected values, $d M$ is absolutely continuous with a density in $L^{2}(d t \otimes d P)$ if and only if $E\left[\int_{-\infty}^{\infty}|\widehat{M}(\theta)|^{2} d \theta\right]$ is finite. To see when the latter happens, let us first rewrite $\widehat{M}$ in a more convenient way. We know, by (7), that the dust's mass can be expressed a.s. as $M(t)=P(I \leqslant t \mid X), t \geqslant 0$, where $I$ corresponds to the first time at which a tagged fragment vanishes. In others words, $d M$ is the conditional law of $I$ given $X$ and $\widehat{M}$ can be written as

$$
\widehat{M}(\theta)=E\left[\mathrm{e}^{i \theta I} \mid X\right], \quad \theta \in \mathbb{R}, \text { a.s. }
$$

Dealing with $|\widehat{M}(\theta)|^{2}$ suggests then to work with two fragments tagged independently. So, consider $U_{1}$ and $U_{2}$, two independent random variables uniformly distributed on $] 0,1[$ and independent of $X$, and the corresponding tagged fragments, as explained in Section 3.2. Let $I_{1}$ (respectively $I_{2}$ ) denote the first time at which the tagged fragment containing $U_{1}$ (respectively $U_{2}$ ) vanishes. These random variables are not independent, however they are independent conditionally on $X$ and then, by (18),

$$
E\left[|\widehat{M}(\theta)|^{2}\right]=E\left[E\left[\mathrm{e}^{i \theta I_{1}} \mid X\right] E\left[\mathrm{e}^{-i \theta I_{2}} \mid X\right]\right]=E\left[\mathrm{e}^{i \theta\left(I_{1}-I_{2}\right)}\right], \quad \theta \in \mathbb{R} .
$$

Recall the notations of Section 3.2: $T_{2}$ is the first time at which the fragments containing the tagged points $U_{1}$ and $U_{2}$ are different and $\lambda_{1}\left(T_{2}\right)$ (respectively $\lambda_{2}\left(T_{2}\right)$ ) the mass of the fragment containing $U_{1}$ (respectively $U_{2}$ ) at that time $T_{2}$. An application of the scaling and strong fragmentation properties at this (randomized) stopping time $T_{2}$ 
leads to the existence of two independent random variables $\widetilde{I}_{1}$ and $\widetilde{I}_{2}$, independent of $\mathcal{F}\left(T_{2}\right)$ and $\left(\lambda_{1}\left(T_{2}\right), \lambda_{2}\left(T_{2}\right)\right)$, and with the same distribution as $I$, such that

$$
I_{1}=T_{2}+\lambda_{1}^{|\alpha|}\left(T_{2}\right) \widetilde{I}_{1} \quad \text { and } \quad I_{2}=T_{2}+\lambda_{2}^{|\alpha|}\left(T_{2}\right) \widetilde{I}_{2}
$$

This yields to

$$
E\left[|\widehat{M}(\theta)|^{2}\right]=E\left[\mathrm{e}^{i \theta\left(\lambda_{1}^{|\alpha|}\left(T_{2}\right) \widetilde{I}_{1}-\lambda_{2}^{|\alpha|}\left(T_{2}\right) \widetilde{I}_{2}\right)}\right]
$$

Our goal is then to show that the characteristic function of the random variable $\lambda_{1}^{|\alpha|}\left(T_{2}\right) \widetilde{I}_{1}-\lambda_{2}^{|\alpha|}\left(T_{2}\right) \widetilde{I}_{2}$ belongs to $L^{1}(d \theta)$.

To prove this, we use the following result (see [12], p. 20): if a function $f \in L^{1}(d x)$, is bounded in a neighborhood of 0 and has a non-negative Fourier transform $\widehat{f}$, then $\widehat{f} \in L^{1}(d x)$. We already know that the characteristic function of $\lambda_{1}^{|\alpha|}\left(T_{2}\right) \widetilde{I}_{1}-\lambda_{2}^{|\alpha|}\left(T_{2}\right) \widetilde{I}_{2}$ is non-negative, since it is equal to $E\left[|\widehat{M}(\theta)|^{2}\right]$. Next, recall that $\widetilde{I}_{1}, \widetilde{I}_{2}$ and $\left(\lambda_{1}\left(T_{2}\right), \lambda_{2}\left(T_{2}\right)\right)$ are independent and that $I$ has a bounded density $k$, according to Lemma 1 and assumptions (A1) and (A2). Let $C$ be an upper bound of $k$. Then, easy calculation shows that the random variable $\lambda_{1}^{|\alpha|}\left(T_{2}\right) \widetilde{I}_{1}-\lambda_{2}^{|\alpha|}\left(T_{2}\right) \widetilde{I}_{2}$ has a density $f$ given by

$$
f(x)=\int_{x \vee 0}^{\infty} E\left[\lambda_{1}^{\alpha}\left(T_{2}\right) \lambda_{2}^{\alpha}\left(T_{2}\right) k\left(u \lambda_{1}^{\alpha}\left(T_{2}\right)\right) k\left((u-x) \lambda_{2}^{\alpha}\left(T_{2}\right)\right)\right] d u, \quad x \in \mathbb{R}
$$

which is bounded by

$$
\begin{aligned}
0 \leqslant f(x) \leqslant & C \int_{x \vee 0}^{\infty} E\left[\lambda_{1}^{\alpha}\left(T_{2}\right) \lambda_{2}^{\alpha}\left(T_{2}\right) k\left((u-x) \lambda_{2}^{\alpha}\left(T_{2}\right)\right) 1_{\left\{\lambda_{1}\left(T_{2}\right) \geqslant \lambda_{2}\left(T_{2}\right)\right\}}\right] d u \\
& +C \int_{x \vee 0}^{\infty} E\left[\lambda_{1}^{\alpha}\left(T_{2}\right) \lambda_{2}^{\alpha}\left(T_{2}\right) k\left(u \lambda_{1}^{\alpha}\left(T_{2}\right)\right) 1_{\left\{\lambda_{2}\left(T_{2}\right) \geqslant \lambda_{1}\left(T_{2}\right)\right\}}\right] d u .
\end{aligned}
$$

The first integral is bounded from above by $E\left[\lambda_{1}^{\alpha}\left(T_{2}\right) 1_{\left\{\lambda_{1}\left(T_{2}\right) \geqslant \lambda_{2}\left(T_{2}\right)\right\}}\right]$ (recall that $\int_{0}^{\infty} k(v) d v=1$ ) and the second one by $E\left[\lambda_{2}^{\alpha}\left(T_{2}\right) 1_{\left\{\lambda_{2}\left(T_{2}\right) \geqslant \lambda_{1}\left(T_{2}\right)\right\}}\right]$. These two expectations are equal. By applying Lemma 2 to $a_{1}=|\alpha|$ and $a_{2}=0$, we see that there are finite as soon as $\alpha>-1$ and $\int_{\mathcal{S} \downarrow} \sum_{i<j} s_{i}^{1+\alpha} s_{j} v(d s)<\infty$. Therefore $f$ is bounded and the function $\theta \in \mathbb{R} \mapsto \widehat{f}(\theta)=E\left[|\widehat{M}(\theta)|^{2}\right]$ belongs to $L^{1}(d \theta)$.

Some examples. Let us now give some examples of fragmentation processes with parameters $\alpha, v$ satisfying assumptions (A1), (A2), such that $\alpha>-1$ and $\int_{\mathcal{S} \downarrow} \sum_{i<j} s_{i}^{1+\alpha} s_{j} v(d s)=\infty$, and such that the mass measure $d M$ does not have a density in $L^{2}(d t \otimes d P)$. Specifically, fix $\alpha>-1$ and consider the dislocation measure

$$
v(d s)=\sum_{n \geqslant 1} a_{n} \delta_{(\underbrace{n^{-1}, n^{-1}, \ldots, n^{-1}}_{n \text { times }}, 0, \ldots)}(d s),
$$

where $\left(a_{n}\right)_{n \geqslant 1}$ is a sequence of non-negative real numbers such that

$$
\sum_{n \geqslant 1} a_{n} \ln n<\infty \quad \text { and } \quad \sum_{n \geqslant 1} a_{n} n^{|\alpha|}=\infty
$$

The assumption $\sum_{n \geqslant 1} a_{n} \ln n<\infty$ leads both to the integrability of $\sum_{i \geqslant 1}\left|\log \left(s_{i}\right)\right| s_{i}$ with respect to $v$ and to the finiteness of $\int_{\mathcal{S} \downarrow}\left(1-s_{1}\right)^{\beta} \nu(d s)$ for $\beta \geqslant 0$. Hence both assumptions (A1) and (A2) are satisfied. The assumption $\sum_{n \geqslant 1} a_{n} n^{|\alpha|}=\infty$ implies $\int_{\mathcal{S} \downarrow} \sum_{i<j} s_{i}^{1+\alpha} s_{j} v(d s)=\infty$ and this in turn will imply that $d M$ has no density in 
$L^{2}(d t \otimes d P)$. To see this, note that the measure $v$ is constructed so that when a fragment splits, it splits into $n$ fragments with same masses for some $1 \leqslant n<\infty$. Combined with (19), this remarks leads to

$$
E\left[|\widehat{M}(\theta)|^{2}\right]=E\left[\mathrm{e}^{i \theta \lambda_{1}^{|\alpha|}\left(T_{2}\right)\left(\widetilde{I}_{1}-\widetilde{I}_{2}\right)}\right]=E\left[\left|\psi_{I}\left(\theta \lambda_{1}^{|\alpha|}\left(T_{2}\right)\right)\right|^{2}\right],
$$

where $\psi_{I}$ denotes the characteristic function of $I$. This characteristic function is in $L^{2}(d x)$, since the density $k$ of the law of $I$ is in $L^{2}(d x)$ (see Lemma 1). Hence $\int_{-\infty}^{\infty} E\left[|\widehat{M}(\theta)|^{2}\right] d \theta$ is finite if and only if $E\left[\lambda_{1}^{\alpha}\left(T_{2}\right)\right]=E\left[\lambda_{1}^{\alpha}\left(T_{2}\right) 1_{\left\{\lambda_{1}\left(T_{2}\right) \geqslant \lambda_{2}\left(T_{2}\right)\right\}}\right]$ is finite. And according to Lemma 2, this last expectation is infinite when $\int_{\mathcal{S} \downarrow} \sum_{i<j} s_{i}^{1+\alpha} s_{j} v(d s)=\infty$, which is the case here. Therefore, $\int_{-\infty}^{\infty} E\left[|\widehat{M}(\theta)|^{2}\right] d \theta$ is infinite and $d M$ cannot be absolutely continuous with a density in $L^{2}(d t \otimes d P)$.

The proof of Theorem 4(ii) relies essentially on the following lemma:

Lemma 6. If $\alpha \leqslant-1$, for a.e. $t$, the number of fragments with positive mass present at time $t$ is finite a.s.

This has already been proved in the last section of [7] for $\alpha<-1$ and extends to $\alpha \leqslant-1$ as follows.

Proof. For fixed time $t$, by applying the fragmentation and scaling properties at that time, we see that we can rewrite the differences $M(t+\varepsilon)-M(t), \varepsilon>0$, as

$$
M(t+\varepsilon)-M(t)=\sum_{i} X_{i}(t) 1_{\left\{X_{i}(t)>0\right\}} M^{(i)}\left(\varepsilon X_{i}(t)^{\alpha}\right), \quad \text { for all } \varepsilon>0,
$$

where the processes $M^{(i)}$ are mutually independent and independent of $\mathcal{F}(t)$, and have the same law as $M$. Let then $\mathcal{T}^{(i)}, i \geqslant 1$, denote the first time at which the dust's mass $M^{(i)}$ reaches 1 and remark that for all $a>0$,

$$
M(t+\varepsilon)-M(t) \geqslant \sum_{i} X_{i}(t) 1_{\left\{0<X_{i}(t)^{|\alpha|} \leqslant \varepsilon / a\right\}} 1_{\left\{\mathcal{T}^{(i)} \leqslant a\right\}}, \quad \varepsilon>0 .
$$

The Lebesgue differentiation theorem implies that a.s., for a.e. $t, \lim _{\varepsilon \rightarrow 0}(M(t+\varepsilon)-M(t)) / \varepsilon$ exists and is finite. By Fubini's theorem, the order of "almost surely" and "for almost every $t$ " can be exchanged and therefore, for a.e. $t$, there exists a finite r.v. $L(t)$ such that

$$
\frac{M(t+\varepsilon)-M(t)}{\varepsilon} \underset{\varepsilon \rightarrow 0}{\stackrel{\text { a.s. }}{\rightarrow}} L(t) .
$$

For such a time $t$, denote by $E_{t}$ the event

"the number of macroscopic fragments at time $t$ is infinite"

and take $\omega$ in $E_{t}$ such that (23) holds. Given a positive $a$, we introduce the (random) sequence $\varepsilon_{n}=a X_{n}(t)(\omega)$. Since $|\alpha| \geqslant 1$ and $\varepsilon_{n}>0$ for all $n \geqslant 1$, we deduce from (22) ( $\omega$ being dropped from notations) that

$$
L(t) \geqslant \frac{1}{a} \lim \sup _{n \rightarrow \infty} \frac{1}{X_{n}(t)} \sum_{i} X_{i}(t) 1_{\left\{0<X_{i}(t) \leqslant X_{n}(t)\right\}} 1_{\left\{\mathcal{T}^{(i)} \leqslant a\right\}} \geqslant \frac{1}{a} \lim _{n \rightarrow \infty} \sup _{\left\{\mathcal{T}^{(n)} \leqslant a\right\}} .
$$

By Lemma 3, $P\left(\mathcal{T}^{(1)} \leqslant a\right)>0$ and then, since the $\mathcal{T}^{(n)}$ are iid,

$$
\lim _{n \rightarrow \infty} \sup _{\left\{\mathcal{T}^{(n)} \leqslant a\right\}}=1 \text { a.s. }
$$

This holds for every $a>0$. In other words, for a.e. $\omega \in E_{t}, L(t)(\omega)=\infty$. But $L(t)<\infty$ a.s, and so $P\left(E_{t}\right)=0$.

Proof of Theorem 4(ii). First, remark that since the first time $I$ at which a tagged fragment vanishes is supposed to satisfy $E\left[I^{-1}\right]<\infty$ and since, by $(7), E[M(\varepsilon)]=P(I \leqslant \varepsilon)$ for $\varepsilon>0$, we have

$$
\frac{E[M(\varepsilon)]}{\varepsilon} \leqslant \int_{0}^{\varepsilon} x^{-1} P(I \in d x) \underset{\varepsilon \rightarrow 0}{\rightarrow} 0 .
$$


Next, choose a time $t$ such that the number of fragments with positive mass present at that time is a.s. finite and remark that the convergence to 0 above combined with formula (21) implies that

$$
E\left[\frac{M(t+\varepsilon)-M(t)}{\varepsilon} \mid \mathcal{F}(t)\right] \underset{\varepsilon \rightarrow 0}{\stackrel{\text { a.s. }}{\rightarrow}} 0 .
$$

Therefore, by Fatou's Lemma,

$$
\lim \inf _{n \rightarrow 0} n(M(t+1 / n)-M(t)) \stackrel{\text { a.s. }}{=} 0 .
$$

According to the previous lemma this holds for a.e $t \geqslant 0$, and we conclude by Fubini's Theorem that a.s., for a.e. $t \geqslant 0, \liminf _{n \rightarrow 0} n(M(t+1 / n)-M(t))=0$. By the Lebesgue differentiability theorem, this is equivalent to the a.s. singularity of $d M$, which ends the proof.

\section{Approximation of the density}

When the mass measure $d M$ of some $(\alpha, v)$-fragmentation $X$ (satisfying hypothesis $(\mathrm{H})$ ) possesses a Lebesgue density, a question that naturally arises, is to know if, as in the Brownian excursion fragmentation discussed in the Introduction, this density can be approximated by functions of small fragments. In most cases, the answer is positive. To see this, introduce for $t \geqslant 0$ and $\varepsilon>0$

$$
M(t, \varepsilon):=\sum_{i \geqslant 1} X_{i}(t) 1_{\left\{0<X_{i}(t) \leqslant \varepsilon\right\}}
$$

the total mass at time $t$ of macroscopic fragments with mass at most $\varepsilon$, and

$$
N(t, \varepsilon):=\sum_{i \geqslant 1} 1_{\left\{X_{i}(t)>\varepsilon\right\}}
$$

the number of fragments present at time $t$ with mass greater than $\varepsilon$. We then have:

Theorem 7. Consider a dislocation measure $v$ such that (A1) holds and suppose that

(a) the mass measure $d M$ is absolutely continuous with a density $L$ in $L^{p}(d x \otimes d P)$ for some $p>1$,

(b) the fragmentation is not geometric, i.e. there exists no $r>0$ such that the mass of every fragment at every time $t$ belongs to the set $\left\{\mathrm{e}^{-k r}: k \in \mathbb{N}\right\}$.

Then, for a.e. $t$,

$$
\varepsilon^{\alpha} M(t, \varepsilon) \underset{\varepsilon \rightarrow 0}{\stackrel{\text { a.s. }}{\rightarrow}} L(t) /|\alpha| \mu
$$

and

$$
\varepsilon^{1+\alpha} N(t, \varepsilon) \underset{\varepsilon \rightarrow 0}{\stackrel{\text { a.s. }}{\rightarrow}} L(t)(1-|\alpha|) /|\alpha|^{2} \mu .
$$

The assumptions (a) and (b) are not so restrictive. First, recall that Theorem 4(i), Section 4, gives sufficient conditions for the mass measure to have a density in $L^{2}(d x \otimes d P)$. Next, concerning assumption (b), it is easy to see that the fragmentation is not geometric as soon as $v\left(\mathcal{S}^{\downarrow}\right)=\infty$. This is a consequence of Corollary 24.6 in [23] and its proof (to see this, consider the subordinator $\xi$ introduced in Section 3 and note that its Lévy measure is finite if and only if $v$ is finite). 
To prove Theorem 7, we need the following lemma and the Wiener-Pitt Tauberian Theorem, which is recalled just after the proof of the lemma.

Lemma 8. Let I be a r.v. independent of $X$, with the same distribution as the first time of vanishing of a tagged fragment (given by (6)). If the mass measure $d M$ is absolutely continuous with a density $L$ in $L^{p}(d x \otimes d P)$ for some $p>1$, then for a.e. $t$,

$$
\lim _{\varepsilon \rightarrow 0} \varepsilon^{\alpha} E\left[M\left(t, \varepsilon I^{-1 /|\alpha|}\right) \mid X\right] \stackrel{\text { a.s. }}{=} L(t) .
$$

Proof. As in the proof of Lemma 6, we rewrite the difference $M(t+\varepsilon)-M(t)$, as

$$
M(t+\varepsilon)-M(t)=\sum_{i} X_{i}(t) 1_{\left\{X_{i}(t)>0\right\}}\left(M^{(i)}\left(\varepsilon X_{i}(t)^{\alpha}\right)\right), \quad \text { for all } \varepsilon>0,
$$

where the processes $M^{(i)}$ are independent copies of $M$ and independent of $\mathcal{F}(t)$. If $I$ denotes a r.v. independent of $X$ and with the same distribution as (6), we get from (7) that $E[M(s)]=P(I \leqslant s)$, for $s \geqslant 0$, and then that

$$
E\left[M^{(i)}\left(\varepsilon X_{i}(t)^{\alpha}\right) \mid \mathcal{F}(t)\right] \stackrel{\text { a.s. }}{=} P\left(I \leqslant \varepsilon X_{i}(t)^{\alpha} \mid \mathcal{F}(t)\right) \stackrel{\text { a.s. }}{=} P\left(I \leqslant \varepsilon X_{i}(t)^{\alpha} \mid X\right), \quad i \geqslant 1 .
$$

Hence, almost surely,

$$
\begin{aligned}
E[M(t+\varepsilon)-M(t) \mid \mathcal{F}(t)] & =\sum_{i} X_{i}(t) 1_{\left\{X_{i}(t)>0\right\}} P\left(I \leqslant \varepsilon X_{i}(t)^{\alpha} \mid X\right) \\
& =E\left[\sum_{i} X_{i}(t) 1_{\left\{0<X_{i}(t)^{|\alpha|} \leqslant \varepsilon I^{-1}\right\}} \mid X\right] \\
& =E\left[M\left(t, \varepsilon^{1 /|\alpha|} I^{-1 /|\alpha|}\right) \mid X\right] .
\end{aligned}
$$

For a.e. $t,(M(t+\varepsilon)-M(t)) / \varepsilon$ converges to $L(t)$ as $\varepsilon \rightarrow 0, L$ being the density of $d M$. Since this density is supposed to belong to $L^{p}(d x \otimes d P)$ for some $p>1$, we may apply the maximal inequality of Hardy-Littlewood (see e.g. [24], p. 5), which yields

$$
\int_{0}^{\infty} \sup _{\varepsilon>0}\left(\frac{M(t)-M(t+\varepsilon)}{\varepsilon}\right)^{p} d t \leqslant C \int_{0}^{\infty} L^{p}(t) d t
$$

for some deterministic constant $C$. Then, for a.e. $t$, the r.v. $\sup _{\varepsilon>0}(M(t+\varepsilon)-M(t)) / \varepsilon$ has a moment of order $p$ and the dominated convergence theorem can be applied in the left-hand side of (26). Therefore, for a.e. $t$,

$$
\lim _{\varepsilon \rightarrow 0} \varepsilon^{\alpha} E\left[M\left(t, \varepsilon I^{-1 /|\alpha|}\right) \mid X\right] \stackrel{\text { a.s. }}{=} E[L(t) \mid \mathcal{F}(t)] \stackrel{\text { a.s. }}{=} L(t),
$$

since $L(t)$ is $\mathcal{F}(t)$-measurable, $\mathcal{F}$ being a right-continuous filtration. This right-continuity of $\mathcal{F}$ is a classical consequence of the Feller property of $X$ (proved in [6]).

The following Wiener-Pitt Tauberian Theorem is proved in [11], on p. 227. We recall that a function $g$ with values in $\mathbb{R}$ is said to be slowly decreasing if

$$
\lim _{\lambda \searrow 1} \lim \inf _{x \rightarrow \infty} \inf _{l \in[1, \lambda]}(g(l x)-g(x)) \geqslant 0 .
$$


Hence a slowly decreasing function is a function whose decrease, if any, is slow. As example, an increasing function is slowly decreasing.

Theorem 9 (Wiener-Pitt). Consider $f, g:(0, \infty) \rightarrow \mathbb{R}$ and let $\check{f}(z):=\int_{0}^{\infty} t^{z} f(1 / t) d t / t$ for $z \in \mathbb{C}$ such that the integral converges. If $\check{f}(z)$ exists and is non-zero for Rez $=0$ and if $g$ is bounded, measurable and slowly decreasing, then

$$
\int_{0}^{\infty} f(x / t) g(t) d t / t \underset{x \rightarrow \infty}{\rightarrow} c \check{f}(0)
$$

implies

$$
g(x) \underset{x \rightarrow \infty}{\rightarrow} c .
$$

By definition, a function $g$ is slowly increasing if $(-g)$ is slowly decreasing. The Wiener-Pitt Theorem thus remains valid for slowly increasing functions $g$.

Proof of Theorem 7. Let us start with the convergence of $\varepsilon^{a} M(t, \varepsilon)$ as $\varepsilon \rightarrow 0$. In that aim, consider $I$ a r.v. independent of $X$ and with the same distribution as the first time of vanishing of a tagged fragment and fix $t \geqslant 0$ such that (24) holds. Then set

$$
f(x):=k(1 / x), \quad x \in(0, \infty)(k \text { is the density of } I)
$$

and

$$
g(x):=x M\left(t, x^{-1 /|\alpha|}\right), \quad x \in(0, \infty)
$$

( $g$ is a random function). The convergence (24) is equivalent to

$$
\int_{0}^{\infty} f(x / u) g(u) d u / u \underset{x \rightarrow \infty}{\stackrel{\text { a.s. }}{\rightarrow}} L(t),
$$

so that, provided that the Wiener-Pitt Theorem applies,

$$
g(x) \underset{x \rightarrow \infty}{\stackrel{\text { a.s. }}{\rightarrow}} L(t) / \check{f}(0) .
$$

This is equivalent to $\varepsilon^{\alpha} M(t, \varepsilon) \underset{\varepsilon \rightarrow 0}{\stackrel{\text { a.s. }}{\rightarrow}} L(t) /|\alpha| \mu$, since $\check{f}(0)=\int_{0}^{\infty} k(t) d t / t=E\left[I^{-1}\right]=|\alpha| \mu$ (by (8)). Thus, we just have to check that $f$ and $g$ satisfy the assumptions of the Wiener-Pitt Theorem.

Consider first $f$. For every $x$ in $\mathbb{R}, \check{f}(i x)=E\left[I^{i x-1}\right]$ exists since $E\left[I^{-1}\right]$ is finite. We would like to show that $E\left[I^{i x-1}\right]$ is non-zero for all $x \in \mathbb{R}$. When $x=0, E\left[I^{-1}\right]>0$ since $I$ is a positive random variable. Now for $x \neq 0$, consider the subordinator $\xi$ introduced in Section 3.2 and related to the law of $I$ by (6). As a consequence of assumption (b), the Lévy measure $\pi_{\alpha}$ of the subordinator $|\alpha| \xi$ is not supported by a set $r \mathbb{N}$, for some $r>0$, so that the characteristic exponent $\psi(x)=\int_{0}^{\infty}\left(1-\mathrm{e}^{i x u}\right) \pi_{\alpha}(d u)$ of this subordinator is non-zero when $x \neq 0$. Then, following the proof of Proposition 3 in [13], we get that $E\left[I^{i x-1}\right]=E\left[I^{i x}\right] \psi(x) / i x$ for $x \neq 0$. Thus we just have to prove that $E\left[I^{i x}\right]$ is non-zero. We know [9] that there exists a random variable $R$, independent of $I$, such that $I R \stackrel{\text { law }}{=} e$ where $e$ denotes the exponential r.v. with parameter 1 . Therefore,

$$
E\left[I^{i x}\right] E\left[R^{i x}\right]=\int_{0}^{\infty} t^{i x} \mathrm{e}^{-t} d t .
$$


This last integral is equal to $\Gamma(1+i x), \Gamma$ being the analytic continuation of the Gamma function, and it is wellknown (see e.g. [2]) that $\Gamma(z) \neq 0$ for all $z$ in the complex plane. Thus $E\left[I^{i x}\right]$ is non-zero.

Now consider the function $g$. Since $x \mapsto M(t, x)$ is non-decreasing, $g$ is bounded from above by

$$
x E\left[M\left(t, x^{-1 /|\alpha|} I^{-1 /|\alpha|}\right) 1_{\{I \leqslant 1\}} \mid X\right] / P(I \leqslant 1),
$$

which is a.s. bounded on $\mathbb{R}_{+}^{*}$ (by (24) and since $P(I \leqslant 1) \geqslant P(\mathcal{T} \leqslant 1)>0$ by Lemma 3 ). The function $x \mapsto M(t, x)$ is a limit of step functions, thus it is measurable and $g$ is measurable. It remains to show that $g$ is slowly increasing, that is

$$
\lim _{\lambda \searrow 1} \lim \inf _{x \rightarrow \infty} \inf _{l \in[1, \lambda]}(g(x)-g(l x)) \geqslant 0 .
$$

We have that

$$
g(x)-g(l x)=x(1-l) M\left(t, x^{-1 /|\alpha|}\right)+l x\left(M\left(t, x^{-1 /|\alpha|}\right)-M\left(t,(l x)^{-1 /|\alpha|}\right)\right) .
$$

For all $l \geqslant 1$, the second term in the right-hand side of this identity is non-negative, which leads to

$$
\inf _{l \in[1, \lambda]}(g(x)-g(l x)) \geqslant(1-\lambda) g(x) .
$$

Now, since $g$ is a.s. bounded, there exists a positive random constant $C$ such that a.s.

$$
\lim \inf _{x \rightarrow \infty} \inf _{l \in[1, \lambda]}(g(x)-g(l x)) \geqslant C(1-\lambda),
$$

and finally,

$$
\lim _{\lambda \searrow 1} \lim \inf _{x \rightarrow \infty} \inf _{l \in[1, \lambda]}(g(x)-g(l x)) \geqslant 0 .
$$

The Wiener-Pitt Theorem therefore applies to $f$ and $g$ and the convergence of $\varepsilon^{\alpha} M(t, \varepsilon)$ to $L(t) /|\alpha| \mu$ as $\varepsilon \rightarrow 0$ is proved.

The last point to show, is the a.s. convergence of $\varepsilon^{1+\alpha} N(t, \varepsilon)$ to $L(t)(1-|\alpha|) /|\alpha|^{2} \mu$ as $\varepsilon \rightarrow 0$. Bertoin's proof, p. 4. in [8], which relies on Abelian-Tauberian theorems, adapts easily here to give

$$
N(t, \varepsilon) \underset{\varepsilon \rightarrow 0}{\sim}\left(\frac{1-|\alpha|}{|\alpha|}\right) \frac{M(t, \varepsilon)}{\varepsilon} .
$$

The asymptotic behavior of $N(t, \varepsilon)$ as $\varepsilon \rightarrow 0$ can then be deduced from that of $M(t, \varepsilon)$.

Some remarks on small fragments behavior. Theorem 7 shows that for most of fragmentations with an index of self-similarity in ]-1, 0[, the small fragments functions $\varepsilon^{\alpha} M(t, \varepsilon)$ and $\varepsilon^{1+\alpha} N(t, \varepsilon)$ converge, for a.e. fixed time $t$, to non-degenerate limits as $\varepsilon \rightarrow 0$. Moreover, for negative-index fragmentations that are not taken into account in Theorem 7, one can $\operatorname{see}^{2}$ that for a.e. $t \geqslant 0, \varepsilon^{\alpha} M(t, \varepsilon)$ and $\varepsilon^{1+\alpha} N(t, \varepsilon)$ are anyway bounded a.s. When $\alpha \leqslant-1$,

${ }^{2}$ With the notations of the proof of Lemma 6 and using (22) and (23), one gets that for a.e. $t$,

$$
\sup _{\varepsilon>0} \frac{1}{\varepsilon} \sum_{i} X_{i}(t) 1_{\left\{0<X_{i}(t)^{|\alpha|} \leqslant \varepsilon / a\right\}} 1_{\left\{\mathcal{T}^{(i)} \leqslant a\right\}} \text { is a.s. finite for all } a>0 .
$$

Consider then $a_{1 / 2}$ such that $P\left(\mathcal{T}^{(1)} \leqslant a_{1 / 2}\right) \geqslant 1 / 2$. Since the r.v. $\mathcal{T}^{(i)}$ are iid and independent of $\mathcal{F}(t)$,

$$
P\left(\sup _{\varepsilon>0} \frac{1}{\varepsilon} \sum_{i} X_{i}(t) 1_{\left\{0<X_{i}(t)^{|\alpha|} \leqslant \varepsilon\right\}} 1_{\left\{\mathcal{T}^{(i)}>a_{1 / 2}\right\}}<\infty\right) \geqslant P\left(\sup _{\varepsilon>0} \frac{1}{\varepsilon} \sum_{i} X_{i}(t) 1_{\left\{0<X_{i}(t)^{|\alpha|} \leqslant \varepsilon\right\}} 1_{\left\{\mathcal{T}^{(i)} \leqslant a_{1 / 2}\right\}}<\infty\right)=1 .
$$

By taking the sum, we see that $\varepsilon^{\alpha} M(t, \varepsilon)$ is a.s. bounded for $t$ such that (23) holds and so does $\varepsilon^{1+\alpha} N(t, \varepsilon)$ in view of equivalence (27). 
we more precisely have that $M(t, \varepsilon)=0$ and $N(t, \varepsilon)$ is constant for $\varepsilon$ small enough, almost surely and for almost every $t$ (it is Lemma 6).

This completes in some way the discussion on the asymptotic behavior of $M(t, \varepsilon)$ and $N(t, \varepsilon)$ as $\varepsilon \rightarrow 0$ undertaken by Bertoin in [8] for fragmentations with a positive index of self-similarity. The investigating methods (and the results) are completely different according whether the index of self-similarity is positive or negative. The positive case relies on a martingale approach (that cannot be shifted to the negative case) and gives, with suitable assumptions on $v$, that

$$
M(t, \varepsilon) \underset{\varepsilon \rightarrow 0}{\stackrel{\text { a.s. }}{\sim}} C(t, \omega) f(\varepsilon) \quad \text { and } \quad N(t, \varepsilon) \underset{\varepsilon \rightarrow 0}{\stackrel{\text { a.s. }}{\sim}} C(t, \omega) C f(\varepsilon) / \varepsilon
$$

for some constants $C(t, \omega), C$ and where $f(\varepsilon)=\int_{\mathcal{S} \downarrow} \sum_{i} s_{i} 1_{\left\{s_{i}<\varepsilon\right\}} v(d s)$. Note that this function depends on $v$ but not on $\alpha$, whereas in the negative case the convergence rate depends only on $\alpha$.

Another remark when $\alpha<0$ and (A1) holds is that the measure $d M$ is singular if and only if $\varepsilon^{\alpha} M(t, \varepsilon) \stackrel{\text { a.s. }}{\rightarrow} 0$ for a.e $t$. To see this, combine Eqs. (22) and (26).

\section{Hausdorff dimension and Hölder-continuity}

When the measure $d M$ is singular, it may be interesting to estimate the "size" of the support of $d M$ (denoted here by $\operatorname{supp}(d M))$, which is the smallest closed set $C$ of $\mathbb{R}_{+}$such that $d M\left(\mathbb{R}_{+} \backslash C\right)=0$. An appropriate concept is then that of Hausdorff dimension:

$$
\operatorname{dim}_{H}(E):=\inf \left\{\gamma>0: m_{\gamma}(E)=0\right\}, \quad E \subset \mathbb{R}_{+},
$$

where

$$
m_{\gamma}(E):=\operatorname{supinf}_{\varepsilon>0} \sum_{i}\left|B_{i}\right|^{\gamma}
$$

the infimum being taken over all collections of intervals with length $\left|B_{i}\right|<\varepsilon$, whose union covers $E$. For background on the subject, see e.g. [14]. In Section 6.1, we give some lower and upper bounds for $\operatorname{dim}_{H}(\operatorname{supp}(d M))$ and $\operatorname{dim}_{H}(d M)$, the latter being defined as

$$
\operatorname{dim}_{H}(d M):=\inf \left\{\operatorname{dim}_{H}(E): d M(E)=1\right\} .
$$

That $\operatorname{dim}_{H}(d M) \leqslant \operatorname{dim}_{H}(\operatorname{supp}(d M))$ holds anyway and we show below that when $\nu\left(\mathcal{S}^{\downarrow}\right)=\infty$ and $\alpha<-1$, these dimensions are different.

It is well known, since the dust's mass $M$ is the distribution function of $d M$, that the Hausdorff dimension of $d M$ is connected to the Hölder-continuity of $M$, in the sense that $\operatorname{dim}_{H}(d M) \geqslant \gamma$ as soon as $M$ is Hölder-continuous of order $\gamma$. Section 6.2 is devoted to this Hölder-continuity of the mass.

For the sequel, we recall that $\rho$ is defined as

$$
\rho=\sup \left\{q: \int_{\mathcal{S} \downarrow} \sum_{i \geqslant 2} s_{i}^{1-q} v(d s)<\infty\right\}
$$

and set

$$
A:=\sup \left\{a \leqslant 1: \int_{\mathcal{S} \downarrow} \sum_{i<j} s_{i}^{1-a} s_{j} v(d s)<\infty\right\} .
$$

Remark that $0 \leqslant \rho \leqslant A \leqslant 1$. 


\subsection{Hausdorff dimensions of $d M$ and $\operatorname{supp}(d M)$}

Recall that $\mathcal{T}$ denotes the first time at which all the initial mass is reduced to dust, so that $\operatorname{supp}(d M) \subset[0, \mathcal{T}]$.

Proposition 10. (i) If (A1) and (A2) hold, then $\operatorname{dim}_{H}(d M) \geqslant 1 \wedge(A /|\alpha|)$ a.s.

(ii) A.s., $\operatorname{dim}_{H}(d M) \leqslant 1 \wedge(1 /|\alpha|)$.

(iii) If $v\left(\mathcal{S}^{\downarrow}\right)<\infty$, then $\operatorname{dim}_{H}(\operatorname{supp}(d M)) \leqslant 1 \wedge(1 /|\alpha|)$ a.s.

(iv) If $v\left(\mathcal{S}^{\downarrow}\right)=\infty$, then the mass $M$ is strictly increasing on $[0, \mathcal{T}]$ and $\operatorname{dim}_{H}(\operatorname{supp}(d M))=1$ a.s.

Let us make two remarks about these results. First, the difference between the above statements (iii) and (iv), can mainly be explained by the Poisson point process construction of homogeneous fragmentations (see [5] and [3]) and the passage from homogeneous to self-similar fragmentations. Indeed, this construction shows that when $v$ is finite the notion of "first splitting" is well-defined and that it occurs at an exponential time $T$ with parameter $v\left(\mathcal{S}^{\downarrow}\right)$, so that $M$ is null near 0 , whereas when $v$ is infinite the splitting times are dense in $\mathbb{R}_{+}$. This will be a key point in the proofs below.

Second, the parameter $A=1$ as soon as $v\left(s_{N+1}>0\right)=0$ for some integer $N$ (this was shown in (16)). Hence in that case, if moreover assumptions (A1) and (A2) hold, the results (i) and (ii) above give

$$
\operatorname{dim}_{H}(d M)=1 \wedge(1 /|\alpha|) \text { a.s. }
$$

We now turn to the proofs. The upper bound stated in Proposition 10(ii) was recently shown in [18] and we refer to this paper for the proof. Concerning statement (i), it is a standard result (see e.g. Theorem 4.13 of Falconer [14]) that the convergence of $\int_{0}^{\infty} \int_{0}^{\infty}|u-v|^{-a} d M(u) d M(v)$ for some real number $a \leqslant 1$ leads to $\operatorname{dim}_{H}(d M) \geqslant a$. Thus, the proof of Proposition 10(i) is an immediate consequence of the following lemma:

Lemma 11. Consider a positive real number a and suppose that assumptions (A1) and (A2) hold. Then

$$
E\left[\int_{0}^{\infty} \int_{0}^{\infty} \frac{d M(u) d M(v)}{|u-v|^{a}}\right]<\infty \Leftrightarrow a<1 \wedge(A /|\alpha|) .
$$

We point out that the implication $\Rightarrow$ does not take into account the assumptions (A1) and (A2).

Proof. Using the same notations as in the proof of Theorem 4(i), we have that

$$
E\left[\int_{0}^{\infty} \int_{0}^{\infty}|u-v|^{-a} d M(u) d M(v)\right]=E\left[\left|I_{1}-I_{2}\right|^{-a}\right]=E\left[\left|\lambda_{1}^{|\alpha|}\left(T_{2}\right) \tilde{I}_{1}-\lambda_{2}^{|\alpha|}\left(T_{2}\right) \tilde{I}_{2}\right|^{-a}\right] .
$$

Suppose first that $a<1 \wedge(A /|\alpha|)$. By assumptions (A1) and (A2) and Lemma 1, we know that $I$ has a density $k$ such that $k(x)$ and $x k(x)$ are bounded on $\mathbb{R}_{+}^{*}$, say by $C$ and $D$, and then that $\lambda_{1}^{|\alpha|}\left(T_{2}\right) \widetilde{I}_{1}-\lambda_{2}^{|\alpha|}\left(T_{2}\right) \widetilde{I}_{2}$ has a density $f$ (see (20) for an explicit expression). Our goal is to prove that $\int_{-\infty}^{\infty}|\theta|^{-a} f(\theta) d \theta$ is finite. From (20), we get that

$$
\begin{aligned}
\int_{0}^{\infty} \theta^{-a} f(\theta) d \theta \leqslant & \int_{0}^{\infty} \theta^{-a} \int_{0}^{\infty} E\left[\lambda_{1}^{\alpha}\left(T_{2}\right) \lambda_{2}^{\alpha}\left(T_{2}\right) k\left((u+\theta) \lambda_{1}^{\alpha}\left(T_{2}\right)\right) k\left(u \lambda_{2}^{\alpha}\left(T_{2}\right)\right) 1_{\left\{\lambda_{1}\left(T_{2}\right) \geqslant \lambda_{2}\left(T_{2}\right)\right\}}\right] d u d \theta \\
& +C \int_{0}^{\infty} \theta^{-a} \int_{\theta}^{\infty} E\left[\lambda_{1}^{\alpha}\left(T_{2}\right) \lambda_{2}^{\alpha}\left(T_{2}\right) k\left(u \lambda_{1}^{\alpha}\left(T_{2}\right)\right) 1_{\left\{\lambda_{2}\left(T_{2}\right) \geqslant \lambda_{1}\left(T_{2}\right)\right\}}\right] d u d \theta .
\end{aligned}
$$


By Fubini's Theorem, the second term in the right-hand side of this inequality is proportional to

$$
\left(\int_{0}^{\infty} u^{1-a} k(u) d u\right) E\left[\lambda_{1}^{|\alpha|(1-a)}\left(T_{2}\right) \lambda_{2}^{\alpha}\left(T_{2}\right) 1_{\left\{\lambda_{2}\left(T_{2}\right) \geqslant \lambda_{1}\left(T_{2}\right)\right\}}\right],
$$

which is finite. Indeed, recall that $I$ has positive moments of all orders and remark that the expectation is bounded from above by $E\left[\lambda_{2}^{\alpha a}\left(T_{2}\right) 1_{\left\{\lambda_{2}\left(T_{2}\right) \geqslant \lambda_{1}\left(T_{2}\right)\right\}}\right]$, which is finite by Lemma 2 , as $a|\alpha|<A \leqslant 1$. Next, in order to bound the first term in the right-hand side of (31), remark that

$$
\int_{0}^{\infty} \theta^{-a} k\left((u+\theta) \lambda_{1}^{\alpha}\left(T_{2}\right)\right) \lambda_{1}^{\alpha}\left(T_{2}\right) d \theta=\left(\lambda_{1}\left(T_{2}\right)\right)^{a \alpha} \int_{0}^{\infty} \theta^{-a} k\left(\theta+u \lambda_{1}^{\alpha}\left(T_{2}\right)\right) d \theta .
$$

Using the upper bounds $C$ of $k(x)$ and $D$ of $x k(x)$, one gets

$$
\int_{0}^{\infty} \theta^{-a} k\left(\theta+u \lambda_{1}^{\alpha}\left(T_{2}\right)\right) d \theta \leqslant C \int_{0}^{1} \theta^{-a} d \theta+D \int_{1}^{\infty} \theta^{-a-1} d \theta<\infty
$$

and so, the first term in the right-hand side of (31) is bounded from above by

$$
E\left[\left(\lambda_{1}\left(T_{2}\right)\right)^{a \alpha} \lambda_{2}^{\alpha}\left(T_{2}\right) 1_{\left\{\lambda_{1}\left(T_{2}\right) \geqslant \lambda_{2}\left(T_{2}\right)\right\}} \int_{0}^{\infty} k\left(u \lambda_{2}^{\alpha}\left(T_{2}\right)\right) d u\right]
$$

multiplied by a finite constant. Since $\lambda_{2}^{\alpha}\left(T_{2}\right) \int_{0}^{\infty} k\left(u \lambda_{2}^{\alpha}\left(T_{2}\right)\right) d u=1$, this expectation is bounded by $E\left[\left(\lambda_{1}\left(T_{2}\right)\right)^{a \alpha} 1_{\left\{\lambda_{1}\left(T_{2}\right) \geqslant \lambda_{2}\left(T_{2}\right)\right\}}\right]$, which is finite, according to Lemma 2 and the assumption on $a$. All this shows that $\int_{0}^{\infty} \theta^{-a} f(\theta) d \theta<\infty$ and then that $\int_{-\infty}^{\infty}|\theta|^{-a} f(\theta) d \theta<\infty$ since the random variable $\lambda_{1}^{|\alpha|}\left(T_{2}\right) \widetilde{I}_{1}-\lambda_{2}^{|\alpha|}\left(T_{2}\right) \widetilde{I}_{2}$ is symmetric.

To prove the converse implication, first note that

$$
\begin{aligned}
E\left[\left|\lambda_{1}^{|\alpha|}\left(T_{2}\right) \widetilde{I}_{1}-\lambda_{2}^{|\alpha|}\left(T_{2}\right) \tilde{I}_{2}\right|^{-a}\right] & \geqslant E\left[1_{\left\{\lambda_{1}\left(T_{2}\right) \geqslant \lambda_{2}\left(T_{2}\right)\right\},\left\{\widetilde{I}_{1} \geqslant \tilde{I}_{2}\right\}}\left|\lambda_{1}^{|\alpha|}\left(T_{2}\right) \widetilde{I}_{1}-\lambda_{2}^{|\alpha|}\left(T_{2}\right) \widetilde{I}_{2}\right|^{-a}\right] \\
& \geqslant E\left[1_{\left\{\lambda_{1}\left(T_{2}\right) \geqslant \lambda_{2}\left(T_{2}\right)\right\}} \lambda_{1}^{a \alpha}\left(T_{2}\right)\right] E\left[1_{\left\{\widetilde{I}_{1} \geqslant \widetilde{I}_{2}\right\}} \widetilde{I}_{1}^{-a}\right],
\end{aligned}
$$

since $\left(\lambda_{1}\left(T_{2}\right), \lambda_{2}\left(T_{2}\right)\right)$ and $\left(\widetilde{I}_{1}, \widetilde{I}_{2}\right)$ are independent. Therefore, by identity (30),

$$
E\left[\int_{0}^{\infty} \int_{0}^{\infty}|u-v|^{-a} d M(u) d M(v)\right]<\infty \Rightarrow E\left[1_{\left\{\lambda_{1}\left(T_{2}\right) \geqslant \lambda_{2}\left(T_{2}\right)\right\}} \lambda_{1}^{a \alpha}\left(T_{2}\right)\right]<\infty,
$$

which is, by Lemma 2 and the definition of $A$, equivalent to $a<(A /|\alpha|)$. On the other hand, one can show that $v \mapsto \int_{0}^{\infty}|u-v|^{-a} d M(u)=\infty$ on

$$
V=\left\{v>0: \limsup _{\varepsilon \rightarrow 0} \varepsilon^{-a}(M(v+\varepsilon)-M(v-\varepsilon))>0\right\}
$$

and the Lebesgue theory implies $d M(V)=1$ when $a \geqslant 1$. Hence $\int_{0}^{\infty} \int_{0}^{\infty}|u-v|^{-a} d M(u) d M(v)=\infty$ when $a \geqslant 1$.

Proof of Proposition 10(iii). Consider an interval representation $F$ of the fragmentation as explained in Section 2 and denote by $\left.\zeta_{x}, x \in\right] 0,1\left[\right.$, the time at which the fragment containing $x$ vanishes, that is $\zeta_{x}=\inf \{t>0: x \notin F(t)\}$. Then set

$$
\mathcal{A}:=\left\{\zeta_{x}, x \in\right] 0,1[\} .
$$


By formula (7), $M(t)=P(I \leqslant t \mid X)$ for all $t \geqslant 0$ a.s., and since $I$ is the first time at which a tagged fragment vanishes, we have $M(t)=\int_{0}^{1} 1_{\left\{\zeta_{x} \leqslant t\right\}} d x, t \geqslant 0$. Then the closure $\overline{\mathcal{A}}$ of $\mathcal{A}$ contains the support of the measure $d M$ and it is sufficient to bound from above $\operatorname{dim}_{H}(\overline{\mathcal{A}})$. Since $v\left(S^{\downarrow}\right)<\infty$, we may consider the first splitting time, denoted by $T$. It is a stopping time. Let $J_{1}, J_{2}, \ldots$ denote the non-empty disjoint intervals obtained after this first split so that $X_{1}(T) \geqslant X_{2}(T) \geqslant \cdots$ are their respective sizes and remark that

$$
\mathcal{A}=\{T\} \bigcup_{i}\left\{\zeta_{x}, x \in J_{i}\right\}
$$

We first need to prove that

$$
\overline{\mathcal{A}}=\{T\} \bigcup_{i} \overline{\left\{\zeta_{x}, x \in J_{i}\right\}}
$$

To that end, take $a$ in $\overline{\bigcup_{i}\left\{\zeta_{x}, x \in J_{i}\right\}}$ and consider a sequence $\left(x_{n}\right)$ in $\bigcup_{i} J_{i}$ such that $\zeta_{x_{n}} \rightarrow a$. Extracting a subsequence if necessary, we may assume that $\left(x_{n}\right)$ converges. Call $x$ its limit and $J_{x_{n}}$ the interval that contains $x_{n}, n \geqslant 1$. Either $\left|J_{x_{n}}\right| \nrightarrow 0$ as $n \rightarrow \infty$ and then there is a subsequence $\left(x_{\varphi(n)}\right)$ such that the number of disjoint $J_{x_{\varphi(n)}}, n \geqslant 1$, is finite, so that there is at least one of these intervals containing an infinite number of $x_{\varphi(n)}$ and then $a \in \bigcup_{i} \overline{\left\{\zeta_{x}, x \in J_{i}\right\}}$. Or, $\left|J_{x_{n}}\right| \rightarrow 0$ as $n \rightarrow \infty$, which implies that $\zeta_{x_{n}} \stackrel{\text { a.s. }}{\rightarrow} T$ as $n \rightarrow \infty$. To see why this last point holds, introduce $\mathcal{T}_{n}$ the first time at which the fragment $J_{x_{n}}$ vanishes during the fragmentation, $n \geqslant 1$. Of course, $T<\zeta_{x_{n}} \leqslant \mathcal{T}_{n}$. By application of the scaling and strong fragmentation properties at time $T$, we see that there exists a r.v. $\mathcal{T}^{(n)}$, independent of $\mathcal{F}(T)$ and with the same distribution as $\mathcal{T}$ (see (10)) such that $\mathcal{T}_{n}-T=\left|J_{x_{n}}\right|^{|\alpha|} \mathcal{T}^{(n)}$. Hence, using that $E[\mathcal{T}]<\infty$ (see Lemma 3 ) and extracting a subsequence if necessary,

$$
0 \leqslant \zeta_{x_{n}}-T \leqslant\left|J_{x_{n}}\right|^{|\alpha|} \mathcal{T}^{(n)} \underset{n \rightarrow \infty}{\rightarrow} 0 \text { a.s. }
$$

So, in both cases, $\overline{\bigcup_{i}\left\{\zeta_{x}, x \in J_{i}\right\}} \subset\{T\} \bigcup_{i} \overline{\left\{\zeta_{x}, x \in J_{i}\right\}}$ and then $\overline{\mathcal{A}} \subset\{T\} \bigcup_{i} \overline{\left\{\zeta_{x}, x \in J_{i}\right\}}$. The opposite inclusion is obvious.

Now, for each $i \geqslant 1$ set $\mathcal{A}_{i}:=\left(\left\{\zeta_{x}, x \in J_{i}\right\}-T\right)\left(X_{i}(T)\right)^{\alpha}$. It follows from the scaling and strong fragmentation properties that the sets $\mathcal{A}_{i}$ are iid with the same law as $\mathcal{A}$ and are independent of $\mathcal{F}(T)$. Combining this with (32) will lead us to $m_{\gamma}(\overline{\mathcal{A}})=0$ for $\gamma>1 /|\alpha|$, which in turn will imply that $\operatorname{dim}_{H}(\overline{\mathcal{A}}) \leqslant 1 /|\alpha|$, by the definitions of $m_{\gamma}$ and $\operatorname{dim}_{H}$ (see respectively (29) and (28)). To see this, fix $\gamma>1 /|\alpha|$ and $\varepsilon>0$ and define for every subset $E$ of $\mathbb{R}_{+}$

$$
m_{\gamma}^{\varepsilon}(E):=\inf _{\begin{array}{c}
\text { coverings of } E \\
\text { by intervals } B_{n} \text { of lengths } \leqslant \varepsilon
\end{array}} \sum_{n}\left|B_{n}\right|^{\gamma} .
$$

Using that

$$
\overline{\mathcal{A}}=\{T\} \bigcup_{i}\left(T+\left(X_{i}(T)\right)^{-\alpha} \overline{\mathcal{A}_{i}}\right)
$$

we have

$$
m_{\gamma}^{\varepsilon}(\overline{\mathcal{A}}) \leqslant \sum_{i}\left(X_{i}(T)\right)^{-\alpha \gamma} m_{\gamma}^{\varepsilon\left(X_{i}(T)\right)^{\alpha}}\left(\overline{\mathcal{A}_{i}}\right) \leqslant \sum_{i}\left(X_{i}(T)\right)^{-\alpha \gamma} m_{\gamma}^{\varepsilon}\left(\overline{\mathcal{A}_{i}}\right) .
$$

Since the first time $\mathcal{T}$ at which all the mass has been reduced to dust has a finite expectation and since $\overline{\mathcal{A}} \subset[0, \mathcal{T}]$, $E\left[\operatorname{mes}_{\gamma}^{\varepsilon}(\overline{\mathcal{A}})\right]$ is finite. Moreover, $\sum_{i}\left(X_{i}(T)\right)=1$ and $X_{1}(T)<1$ a.s., which implies that $E\left[\sum_{i}\left(X_{i}(T)\right)^{-\alpha \gamma}\right]<1$ when $\gamma>1 /|\alpha|$. Combining this with (33) and the fact that the random variables $\overline{\mathcal{A}_{i}}$ are independent of $\mathcal{F}(T)$ and have the same law as $\overline{\mathcal{A}}$ implies that $E\left[\operatorname{mes}_{\gamma}^{\varepsilon}(\overline{\mathcal{A}})\right]=0$ for all positive $\varepsilon$ as soon as $\gamma>1 /|\alpha|$. So by definition, $m_{\gamma}(\overline{\mathcal{A}}) \stackrel{\text { a.s. }}{=} 0$ for $\gamma>1 /|\alpha|$ and then $\operatorname{dim}_{H}(\overline{\mathcal{A}}) \leqslant 1 /|\alpha|$ a.s. 
Proof of Proposition 10(iv). We first prove that $P(M(t)=0)=0$ for all $t>0$. To do so, fix $t>0$ and take $s$ such that $0<s<t$. Recall that the fragmentation and scaling properties applied at time $s$ give

$$
M(t)=M(s)+\sum_{i} X_{i}(s) 1_{\left\{X_{i}(s)>0\right\}} M^{(i)}\left((t-s) X_{i}^{\alpha}(s)\right)
$$

where the $M^{(i)}$ are mutually independent, independent of $\mathcal{F}(s)$ and with the same distribution as $M$. Since $v\left(\mathcal{S}^{\downarrow}\right)=\infty$, the number of splits before time $s$ is almost surely infinite. So if $M(s)=0$, that is no mass is lost at time $s$, none of the fragments with positive mass appeared before $s$ has entirely vanished at time $s$, so that there is an infinite number of fragments with positive mass present at time $s$. In particular, if $M(t)=0$, then $M(s)=0$ and $X_{i}(s)>0$ for all $i \geqslant 1$. This gives with (34) that when $M(t)=0$, then $M^{(i)}\left((t-s) X_{i}^{\alpha}(s)\right)=0$ and $X_{i}^{\alpha}(s) \nearrow_{i \rightarrow \infty} \infty$. But this event has probability 0 since $P(M(u)=0)<1$ for some $u$ large enough. Therefore, $P(M(t)=0)=0$ and this holds for all $t>0$.

Next, take again $0<s<t$. The mass $M^{(1)}$ being that introduced in (34), remark that conditionally on $X_{1}(s)>0$, we have that $1_{\left\{X_{1}(s)>0\right\}} M^{(1)}\left((t-s) X_{1}^{\alpha}(s)\right)>0$ a.s. since we have just proved that $P(M(u)>0)=1$ for all $u>0$. Hence, by (34), $M(t)>M(s)$ a.s. conditionally on $X_{1}(s)>0$. In others words, $P(M(s)<M(t) \mid s<\mathcal{T})=1$. Since this holds for all $0<s<t$ and since the dust's mass $M$ is a non-decreasing function,

$$
P(M(s)<M(t) \text { for all } 0 \leqslant s<t \leqslant \mathcal{T})=1 .
$$

Hence $M$ is a.s. strictly increasing on $[0, \mathcal{T}]$ and $\operatorname{supp}(d M)=[0, \mathcal{T}]$.

\subsection{Hölder continuity of the dust's mass $M$}

Notice that Proposition 10(ii) implies that a.s. $M$ cannot be Hölder continuous of order $\gamma>1 \wedge(1 /|\alpha|)$, since the $\gamma$-Hölder-continuity of $M$ yields to $\operatorname{dim}_{H}(d M) \geqslant \gamma$ (see Section 13.7 in [14]). We have moreover:

Proposition 12. Suppose that assumptions (A1) and (A2) hold. Then,

(i) the mass $M$ is a.s. Hölder-continuous of order $\gamma$ for every $\gamma<(1 / 2) \wedge(A / 2|\alpha|)$;

(ii) if $v\left(s_{N+1}>0\right)=0$ for some integer $N$, the mass $M$ is a.s. Hölder-continuous of order $\gamma$ for every $\gamma<1 \wedge(\rho /|\alpha|)$.

The upper bound $1 \wedge(\rho /|\alpha|)$ is larger than $(1 / 2) \wedge(A / 2|\alpha|)$ as soon as $\rho \geqslant A / 2$ or $|\alpha| \leqslant 2 \rho$. Remark also that when $v\left(s_{N+1}>0\right)=0$ for some integer $N$, the coefficient $A=1$ (see (16)) and the coefficient $\rho=1$ if and only if $v$ is moreover finite.

Part (i) of Proposition 12 is just a consequence of Lemma 11:

Proof of Proposition 12(i). Consider $\gamma \in] 0,1 \wedge(A /|\alpha|)[$ and remark that for all $t>s \geqslant 0$,

$$
(M(t)-M(s))^{2}=\int_{s}^{t} \int_{s}^{t} d M(u) d M(v) \leqslant(t-s)^{\gamma} \int_{s}^{t} \int_{s}^{t} \frac{d M(u) d M(v)}{|u-v|^{\gamma}} .
$$

The integral $\int_{0}^{\infty} \int_{0}^{\infty}|u-v|^{-\gamma} d M(u) d M(v)$ is a.s. finite by Lemma 11, and then,

$$
|M(t)-M(s)| \leqslant B(t-s)^{\gamma / 2} \text { for all } t>s \geqslant 0
$$

for some a.s. finite constant $B$.

The proof of the second part of Proposition 12 is slightly longer. The point is to use the well-known Kolmogorov criterion (see e.g. [22], p. 26, Theorem 2.1). In that aim, we first prove the following lemma. 
Lemma 13. Suppose that there exists an integer $N$ such that $v\left(s_{N+1}>0\right)=0$ and fix an integer $n \geqslant 2$. Suppose moreover that for all $k \in\{1, \ldots, n-1\}$ there exist a finite constant $C_{k}$ and a positive real number $a_{k}<k \wedge((k-1+\rho) /|\alpha|)$ such that

$$
E\left[(M(t)-M(s))^{k}\right] \leqslant C_{k}(t-s)^{a_{k}} \text { for all } t \geqslant s \geqslant 0 .
$$

Then, for all $a<\inf _{n_{1}+n_{2}+\cdots+n_{l}=n}\left(a_{n_{1}}+\cdots+a_{n_{l}}\right) \wedge((n-1) /|\alpha|)$, there exists a finite constant $C_{n, a}$ such that

$$
E\left[(M(t)-M(s))^{n}\right] \leqslant C_{n, a}(t-s)^{a} \quad \text { for all } t \geqslant s \geqslant 0 .
$$

Proof. Consider $n$ points tagged independently, as explained in Section 3.2, and denote by $I_{1}, \ldots, I_{n}$ their respective times of reduction to dust. The r.v. $I_{i}, 1 \leqslant i \leqslant n$, have the same distribution as $I$ (see (6)). By construction, the $I_{i}$ 's are independent conditionally on $X$, and therefore, by formula (7), we have that

$$
E\left[\prod_{i=1}^{n} 1_{\left\{s<I_{i} \leqslant t\right\}}\right]=E\left[(M(t)-M(s))^{n}\right] .
$$

As in the proof of Theorem 4(i), the goal is now to "introduce some independence" in order to bound from above this expectation. To that end, consider $T_{n}$, the first time at which the $n$ tagged points do not belong to the same fragment and consider the distribution of the tagged points at that time. More precisely, for each integer $l \geqslant 2$ and each $l$-tuple $\left(n_{1}, n_{2}, \ldots, n_{l}\right) \in(\mathbb{N} \backslash\{0\})^{l}$ satisfying $n_{1}+n_{2}+\cdots+n_{l}=n$, consider the event

$$
A_{\left(n_{1}, \ldots, n_{l}\right)}=\left\{\begin{array}{l}
U_{1}, U_{2}, \ldots, U_{l} \text { belong all to different fragments at time } T_{n} \text { and there } \\
\text { are } n_{k} \text { tagged points in the fragment containing } U_{k}, 1 \leqslant k \leqslant l
\end{array}\right\} .
$$

Since the number of such events is finite and since the law of $\left(I_{1}, \ldots, I_{n}\right)$ is exchangeable, we just have to prove that for a fixed $l$-tuple $\left(n_{1}, n_{2}, \ldots, n_{l}\right)$ and all $a<\left(a_{n_{1}}+\cdots+a_{n_{l}}\right) \wedge(n-1) /|\alpha|$, there exists a finite constant $C$ such that

$$
E\left[\prod_{i=1}^{n} 1_{\left\{s<I_{i} \leqslant t\right\}} 1_{\left\{A_{\left(n_{1}, n_{2}, \ldots, n_{l}\right)}\right\}}\right] \leqslant C(t-s)^{a} \quad \text { for all } t \geqslant s \geqslant 0 .
$$

Conditionally on $A_{\left(n_{1}, n_{2}, \ldots, n_{l}\right)}$, there are $l$ tagged fragments at time $T_{n}$, with respective masses, $\lambda_{1}\left(T_{n}\right), \ldots, \lambda_{l}\left(T_{n}\right)$ and containing each, respectively, $n_{1}, \ldots, n_{l}$ tagged points. Write then

$$
\prod_{i=1}^{n} 1_{\left\{s<I_{i} \leqslant t\right\}} 1_{\left\{A_{\left(n_{1}, n_{2}, \ldots, n_{l}\right)}\right\}}=\prod_{k=1}^{l} \prod_{\begin{array}{c}
i: U_{i}, U_{k} \in \operatorname{same} \\
\text { fragment at time } T_{n}
\end{array}} 1_{\left\{s<I_{i} \leqslant t\right\}} 1_{\left\{A_{\left(n_{1}, n_{2}, \ldots, n_{l}\right)}\right\}}
$$

and recall that the $l$ fragments evolve independently after time $T_{n}$. Recall also the scaling property of the fragmentation and consider the identity (36) (that holds for every integer $n$, and in particular the $n_{k}$ 's). Then, setting $M(t):=0$ for $t<0$, there exists a random process $\widetilde{M}$ with the same law as $M$ and independent of $\mathcal{F}\left(T_{n}\right),\left(\lambda_{1}\left(T_{n}\right), \ldots, \lambda_{n}\left(T_{n}\right)\right)$ and $A_{\left(n_{1}, n_{2}, \ldots, n_{l}\right)}$ such that

$$
\begin{aligned}
E[ & \left.\prod_{i=1}^{n} 1_{\left\{s<I_{i} \leqslant t\right\}} 1_{\left\{A_{\left(n_{1}, n_{2}, \ldots, n_{l}\right)}\right\}}\right] \\
= & E\left[\prod_{k=1}^{l} E\left[\left(\tilde{M}\left(\left(t-T_{n}\right) \lambda_{k}^{\alpha}\left(T_{n}\right)\right)-\tilde{M}\left(\left(s-T_{n}\right) \lambda_{k}^{\alpha}\left(T_{n}\right)\right)\right)^{n_{k}} \mid \mathcal{F}\left(T_{n}\right), \lambda_{1}\left(T_{n}\right), \ldots, \lambda_{l}\left(T_{n}\right), A_{\left(n_{1}, n_{2}, \ldots, n_{l}\right)}\right]\right. \\
& \left.\times 1_{\left\{A_{\left(n_{1}, n_{2}, \ldots, n_{l}\right)}\right\}}\right] .
\end{aligned}
$$


Now consider the assumptions we have made in the statement. Since $M$ is a.s. bounded by 1 , the inequality (35) holds actually by replacing $a_{n_{k}}$ by any $b_{n_{k}} \leqslant a_{n_{k}}$ and $C_{n_{k}}$ by $C_{n_{k}} \vee 1$. Therefore, for each $l$-tuple $\left(b_{n_{1}}, \ldots, b_{n_{l}}\right)$ such that $b_{n_{k}} \leqslant a_{n_{k}}, 1 \leqslant k \leqslant l$, there exists a finite deterministic constant $C$ such that

$$
\begin{aligned}
& \prod_{k=1}^{l}\left[E\left(\tilde{M}\left(\left(t-T_{n}\right) \lambda_{k}^{\alpha}\left(T_{n}\right)\right)-\tilde{M}\left(\left(s-T_{n}\right) \lambda_{k}^{\alpha}\left(T_{n}\right)\right)\right)^{n_{k}} \mid \mathcal{F}\left(T_{n}\right), \lambda_{1}\left(T_{n}\right), \ldots, \lambda_{l}\left(T_{n}\right), A_{\left(n_{1}, n_{2}, \ldots, n_{l}\right)}\right] \\
& \quad \leq C(t-s)^{b_{n_{1}}+\cdots+b_{n_{l}}} \prod_{k=1}^{l} \lambda_{k}^{\alpha b_{n_{k}}}\left(T_{n}\right) .
\end{aligned}
$$

And then

$$
E\left[\prod_{i=1}^{n} 1_{\left\{s<I_{i} \leqslant t\right\}} 1_{\left\{A_{n_{1}, n_{2}, \ldots, n_{l}}\right\}}\right] \leqslant C(t-s)^{b_{n_{1}}+\cdots+b_{n_{l}}} E\left[\prod_{k=1}^{l} \lambda_{k}^{\alpha b_{n_{k}}}\left(T_{n}\right) 1_{\left\{A_{\left(n_{1}, n_{2}, \ldots, n_{l}\right)}\right\}}\right] .
$$

To see when the latter expectation is finite we use Lemma 2. Since, by assumption, $v\left(s_{N+1}>0\right)=0$ and $|\alpha| b_{n_{k}}<n_{k}$ (recall that $\rho \leqslant 1$ ) for $1 \leqslant k \leqslant l$,

$$
\int_{\mathcal{S} \downarrow} \sum_{1 \leqslant i_{1}<\cdots<i_{l} \leqslant N} \prod_{k=1}^{l} s_{i_{k}}^{n_{k}-|\alpha| b_{n_{k}}} 1_{\left\{s_{i_{k}}>0\right\}} v(d s) \leqslant N^{l-1} \int_{\mathcal{S} \downarrow} \sum_{2 \leqslant i_{2} \leqslant N} s_{i_{2}}^{n_{2}+\cdots+n_{l}-|\alpha| b_{n_{2}}-\cdots-|\alpha| b_{n_{l}}} v(d s),
$$

which is finite, by definition of $\rho$, as soon as $n_{2}+\cdots+n_{l}-|\alpha| b_{n_{2}}-\cdots-|\alpha| b_{n_{l}}>1-\rho$. This holds here since $|\alpha| b_{n_{k}}<n_{k}-1+\rho$ for $k \geqslant 2$. Thus, by Lemma 2 ,

$$
E\left[\prod_{k=1}^{l} \lambda_{k}^{\alpha b_{n_{k}}}\left(T_{n}\right) 1_{\left\{\lambda_{1}\left(T_{n}\right) \geqslant \lambda_{2}\left(T_{n}\right) \geqslant \cdots \geqslant \lambda_{l}\left(T_{n}\right)\right\}} 1_{\left\{A_{\left(n_{1}, n_{2}, \ldots, n_{l}\right)}\right.}\right]<\infty
$$

as soon as $\sum_{k=1}^{l} b_{n_{k}}<(n-1) /|\alpha|$. By exchangeability, the expectation in the right hand side of inequality (38) is then finite and thus the upper bound (37) and the required result are proved.

Proof of Proposition 12(ii). For all integer $n \geqslant 1$, define

$$
\gamma_{n}:=\sup \left\{a \geqslant 0: \exists C<\infty \text { such that } E\left[(M(t)-M(s))^{n}\right] \leqslant C(t-s)^{a} \text { for all } t \geqslant s \geqslant 0\right\} .
$$

It is well-defined since $M$ is a.s. bounded by 1 . Our goal is to prove that the claim

$$
C(k): \quad \gamma_{n} \geqslant n\left(\frac{k-1}{k} \wedge \frac{\rho}{|\alpha|} \wedge \frac{k-1}{k|\alpha|}\right) \text { for all } n \geqslant 1,
$$

holds for all integers $k \geqslant 1$. If this is true, the proof is finished, since the Kolmogorov criterion then asserts that for each $k \geqslant 1$ and every $\gamma$ such that

$$
\gamma<\left(\frac{k-1}{k} \wedge \frac{\rho}{|\alpha|} \wedge \frac{k-1}{k|\alpha|}\right)-\frac{1}{k}
$$

there is a $\gamma$-Hölder-continuous version of $M$. Since $M$ is non-decreasing, it is actually $M$ that is a.s. Höldercontinuous with these orders $\gamma$. Letting $k \rightarrow \infty, M$ is then a.s. $\gamma$-Hölder-continuous for every $\gamma<(\rho /|\alpha|) \wedge 1$.

So let us prove by induction the claims $C(k), k \geqslant 1$. That $C(1)$ holds is obvious. To prove $C(2)$, remark first that $\gamma_{1}=1$. This is a consequence of formula (7), which gives $E[M(t)-M(s)]=E\left[1_{\{s<I<t\}}\right]$ and then of assumptions (A1) and (A2), which, by Lemma 1, imply that $I$ has a bounded density. Then, $\gamma_{1}=1$ and Lemma 13 lead to $\gamma_{2} \geqslant 2(1 \wedge((\rho \wedge 1 / 2) /|\alpha|))$. And next, using recursively the same lemma and the fact that $\rho \leqslant 1$, we get that

$$
\gamma_{n} \geqslant n(1 \wedge(\rho /|\alpha|) \wedge(1 / 2|\alpha|)) \text { for all } n \geqslant 1 \text {. }
$$


Which proves the claim $C(2)$. Fix now an integer $k \geqslant 2$ and suppose that $C(k)$ holds. We want to prove $C(k+1)$. By Hölder's inequality,

$$
E\left[(M(t)-M(s))^{k+1}\right] \leqslant E\left[(M(t)-M(s))^{k n}\right]^{1 / n} E\left[(M(t)-M(s))^{n /(n-1)}\right]^{(n-1) / n} .
$$

First, remark the existence of a finite constant $C$ such that $E\left[(M(t)-M(s))^{n /(n-1)}\right] \leqslant C(t-s)$ since $0 \leqslant$ $M(t)-M(s) \leqslant 1$ for $t \geqslant s$ and since $I$ has a bounded density. Next, by claim $C(k)$,

$$
\gamma_{n k} \geqslant n((k-1) \wedge(k \rho /|\alpha|) \wedge((k-1) /|\alpha|)) \text { for all } n \geqslant 1,
$$

and this implies, with the previous remark and (39), that

$$
\gamma_{k+1} \geqslant(k-1) \wedge(k \rho /|\alpha|) \wedge((k-1) /|\alpha|)+(n-1) / n \text { for all } n \geqslant 1 .
$$

Letting $n \rightarrow \infty$ and using that $k-1>0$, it is easy to see that

$$
\gamma_{k+1} \geqslant(k-1) \wedge(k \rho /|\alpha|) \wedge((k-1) /|\alpha|)+1 \geqslant k \wedge((k+1) \rho /|\alpha|) \wedge(k /|\alpha|) .
$$

When $n \leqslant k+1$,

$$
E\left[(M(t)-M(s))^{n}\right] \leqslant E\left[(M(t)-M(s))^{(k+1)}\right]^{n /(k+1)}
$$

and then $\gamma_{n} \geqslant n \gamma_{k+1} /(k+1)$. Hence,

$$
\gamma_{n} \geqslant n(k \wedge((k+1) \rho /|\alpha|) \wedge(k /|\alpha|)) /(k+1) \quad \text { for all } n \leqslant k+1 .
$$

Next, by applying Lemma 13 recursively, we get that

$$
\gamma_{n} \geqslant n(k \wedge((k+1) \rho /|\alpha|) \wedge(k /|\alpha|)) /(k+1) \text { for } n>k+1
$$

and so $C(k+1)$ holds. Hence the claims $C(k)$ hold for every integers $k \geqslant 1$.

\section{Acknowledgement}

I am very grateful to Jean Bertoin for helpful discussions and for reading numerous versions of this manuscript.

\section{Appendix A. Proof of Lemma 2}

For this technical proof, it is easier to work with partition-valued fragmentations, so we first recall some background on the subject. The following recalls hold for any self-similar fragmentation. We refer to $[3,5,6]$ for details.

Define by $\mathcal{P}$ the set of partitions of $\mathbb{N} \backslash\{0\}$ and for $\pi \in \mathcal{P}$ and $i \in \mathbb{N} \backslash\{0\}$, denote by $\pi_{i}$ the block of $\pi$ having $i$ as least element, when such a block exists, and set $\pi_{i}:=\emptyset$ otherwise, so that $\left(\pi_{1}, \pi_{2}, \ldots\right)$ are the blocks of $\pi$. A random partition is called exchangeable if its distribution is invariant under finite permutations. Kingman [20] shows that the blocks of every exchangeable partition $\pi$ have asymptotics frequencies a.s., that is (\# denoting the counting measure on $\mathbb{N} \backslash\{0\})$ :

$$
\lim _{n \rightarrow \infty} \frac{\#\left(\pi_{i} \cap\{1, \ldots, n\}\right)}{n} \text { exists a.s. for all } i \text {. }
$$

Let $|\pi|^{\downarrow}$ denote the decreasing rearrangement of these limits.

Now, let $X$ be a $\mathcal{S}^{\downarrow}$-valued fragmentation with index of self-similarity $\alpha$ and consider $F$, one of its interval representation as explained in Section 2. By picking independent r.v. $U_{i}, i \geqslant 1$, uniformly distributed on ]0, 1[ and independent of $F$, we can construct an $\alpha$-self-similar partition-valued fragmentation $(\Pi(t), t \geqslant 0)$ as follows: 
for each $t \geqslant 0, \Pi(t)$ is the random partition of $\mathbb{N} \backslash\{0\}$ such that two integers $i, j$ belong to the same block of $\Pi(t)$ if and only if $U_{i}$ and $U_{j}$ belong to the same interval component of $F(t)$. If $U_{i} \notin F(t)$, then the block of $\Pi(t)$ containing $i$ is $\{i\}$. This process $\Pi$ is exchangeable and called partition-valued representation of $X$. By the strong law of large number, the law of $X$ can be recovered from $\Pi$, as the law of the decreasing rearrangement of asymptotics frequencies of $\Pi$ :

$$
\left(|\Pi(t)|^{\downarrow}, t \geqslant 0\right) \stackrel{\text { law }}{=} X .
$$

In the homogeneous case $(\alpha=0)$, the partition-valued fragmentation $(\Pi(t), t \geqslant 0)$ can be constructed from a Poisson point process (PPP) with an intensity measure depending on the dislocation measure $v$. We explain the construction for a fragmentation with no erosion and a dislocation measure $v$ such that $v\left(\sum_{i} s_{i}<1\right)=0$. First, for every $s=\left(s_{1}, s_{2}, \ldots\right) \in \mathcal{S}^{\downarrow}$, consider the paintbox partition $\Pi_{s}$ (introduced by Kingman, see e.g. [20]) defined as follows: let $\left(Z_{i}\right)_{i} \geqslant 1$ be an iid sequence of random variable such that $P\left(Z_{1}=j\right)=s_{j}$ for $j \geqslant 1$ and let then $\Pi_{s}$ be the partition such that two integers $i, j$ are in the same block if and only if $Z_{i}=Z_{j}$. Introduce next the measure $\kappa_{v}$ defined by

$$
\kappa_{\nu}(B)=\int_{\mathcal{S} \downarrow} P\left(\Pi_{s} \in B\right) v(d s), \quad B \in \mathcal{P} .
$$

Bertoin [5] shows that $\kappa_{v}$ is an exchangeable measure and that the fragmentation $\Pi$ is a pure jumps process whose jumps correspond to the atoms of a PPP $((\Delta(t), k(t)), t \geqslant 0)$ on $\mathcal{P} \times \mathbb{N} \backslash\{0\}$ with intensity $\kappa_{v} \otimes \#$. By this, we mean that $\Pi$ jumps exactly at the times of occurrence of atoms of the PPP and that at such times $t, \Pi(t-)$ jumps to $\Pi(t)$ as follows: the blocks of $\Pi(t)$ are the same as those of $\Pi(t-)$, except $\Pi(t-)_{k(t)}$, which is replaced by the blocks $\left\{n_{i}: i \in \Delta(t)_{1}\right\},\left\{n_{i}: i \in \Delta(t)_{2}\right\}, \ldots$ where $n_{1}<n_{2}<\cdots$ are the elements of the block $\Pi(t-)_{k(t)}$. Berestycki adapts in [3] this PPP-construction to homogeneous $\mathcal{S}^{\downarrow}$-valued fragmentations.

This partition point of view and the Poissonian construction lead to the following lemma.

Lemma 14. Let $F_{h}$ be a homogeneous interval fragmentation, with no erosion and with a dislocation measure $v$ such that $v\left(\sum_{i} s_{i}<1\right)=0$. In this fragmentation, tag independently $n$ fragments as explained in Section 3.2 and let $U_{1, h}, \ldots, U_{n, h}$ denote the tagged points. Define $\lambda_{1, h}(t), \ldots, \lambda_{n, h}(t)$ to be the masses at time $t$ of these tagged fragments and $T_{n, h}$ the first time at which the tagged points do not all belong to the same fragment. For every l-tuple $\left(n_{1}, n_{2}, \ldots, n_{l}\right) \in(\mathbb{N} \backslash\{0\})^{l}$ such that $n_{1}+n_{2}+\cdots+n_{l}=n$, define then $A_{\left(n_{1}, \ldots, n_{l}\right), h}$ by

$$
A_{\left(n_{1}, \ldots, n_{l}\right), h}:=\left\{\begin{array}{l}
U_{1, h}, U_{2, h}, \ldots, U_{l, h} \text { belong all to different fragments at time } T_{n, h} \text { and } \\
\text { there are } n_{k} \text { tagged points in the fragment containing } U_{k, h}, 1 \leqslant k \leqslant l
\end{array}\right\} .
$$

Then,

(i) $\lambda_{1, h}\left(T_{n, h}-\right)=\lambda_{2, h}\left(T_{n, h}-\right)=\cdots=\lambda_{n, h}\left(T_{n, h}-\right)$ by definition of $T_{n, h}$,

(ii) $A_{\left(n_{1}, \ldots, n_{l}\right), h}$ and $\left(\frac{\lambda_{1, h}\left(T_{n, h}\right)}{\lambda_{1, h}\left(T_{n, h}-\right)}, \frac{\lambda_{2, h}\left(T_{n, h}\right)}{\lambda_{1, h}\left(T_{n, h}-\right)}, \ldots, \frac{\lambda_{n, h}\left(T_{n, h}\right)}{\lambda_{1, h}\left(T_{n, h}-\right)}\right)$ are independent of $\lambda_{1, h}\left(T_{n, h}-\right)$,

(iii) there is a positive finite constant $C$ such that for every positive measurable function $f$ on $] 0,1]^{l}$,

$$
\begin{array}{r}
E\left[f\left(\frac{\lambda_{1, h}\left(T_{n, h}\right)}{\lambda_{1, h}\left(T_{n, h}-\right)}, \frac{\lambda_{2, h}\left(T_{n, h}\right)}{\lambda_{1, h}\left(T_{n, h}-\right)}, \ldots, \frac{\lambda_{l, h}\left(T_{n, h}\right)}{\lambda_{1, h}\left(T_{n, h}-\right)}\right) 1_{\left\{A_{\left(n_{1}, n_{2}, \ldots, n_{l}\right), h}\right\}}\right] \\
=C \int_{\mathcal{S} \downarrow} \sum_{i_{1} \neq i_{2} \neq \cdots \neq i_{l}} s_{i_{1}}^{n_{1}} s_{i_{2}}^{n_{2}} \ldots s_{i_{l}}^{n_{l}} f\left(s_{i_{1}}, \ldots, s_{i_{l}}\right) 1_{\left\{s_{i_{1}}>0, \ldots, s_{i_{l}}>0\right\}} v(d s) .
\end{array}
$$

Proof. Let $\left(\Pi_{h}(t), t \geqslant 0\right)$ be the homogeneous partition-valued fragmentation constructed from $F_{h}$ and the $U_{i, h}$ 's, and let $((\Delta(t), k(t)), t \geqslant 0)$ be the PPP on $\mathcal{P} \times \mathbb{N} \backslash\{0\}$ with intensity $\kappa_{v} \otimes \#$ describing the jumps of $\Pi_{h}$. Define then 
$\mathcal{P}_{n}^{*}$ to be the set of partitions of $\mathbb{N} \backslash\{0\}$ such that integers $1,2, \ldots, n$ do not belong to the same block and remark that

$$
T_{n, h}=\inf \left\{t \geqslant 0: \Pi_{h}(t) \in \mathcal{P}_{n}^{*}\right\}=\inf \left\{t \geqslant 0: \Delta(t) \in \mathcal{P}_{n}^{*} \text { and } k(t)=1\right\} .
$$

Setting $\Delta_{i}$ for the block of $\Delta\left(T_{n, h}\right)$ containing $i, 1 \leqslant i \leqslant n$, the event $A_{\left(n_{1}, n_{2}, \ldots, n_{l}\right), h}$ can therefore be written as

$$
A_{\left(n_{1}, n_{2}, \ldots, n_{l}\right), h}=\left\{\begin{array}{l}
1,2, \ldots, l \text { belong to distinct blocks of } \Delta\left(T_{n, h}\right) \\
\text { and } \operatorname{Card}\left(\Delta_{k} \cap\{1, \ldots, n\}\right)=n_{k}, 1 \leqslant k \leqslant l
\end{array}\right\} .
$$

and using the exchangeability of $\kappa_{\nu}$ and the independence of $\Delta\left(T_{n, h}\right)$ and $\Pi_{h}\left(T_{n, h}-\right)$, we get that

$$
\frac{\#\left(\Delta_{i} \cap\{1, \ldots, k\}\right)}{k} \underset{k \rightarrow \infty}{\stackrel{\text { a.s. }}{\rightarrow}} \frac{\lambda_{i, h}\left(T_{n, h}\right)}{\lambda_{1, h}\left(T_{n, h}-\right)}, \quad 1 \leqslant i \leqslant n,
$$

and then assertion (ii).

Next, to prove (iii), note that formula (A.1) leads to

$$
\kappa_{\nu}\left(\mathcal{P}_{n}^{*}\right)=\int_{\mathcal{S} \downarrow}\left(1-\sum_{i} s_{i}^{n}\right) v(d s)
$$

which is positive and finite since $1-\sum_{i} s_{i}^{n} \leqslant n\left(1-s_{1}\right)$ and $\left(1-s_{1}\right)$ is integrable with respect to $v$. It is then a standard result of PPP's theory that $T_{n, h}$ has an exponential law with parameter $\kappa_{v}\left(\mathcal{P}_{n}^{*}\right)$ and that the distribution of $\Delta\left(T_{n, h}\right)$ is given by $\kappa_{v}\left(\cdot \bigcap \mathcal{P}_{n}^{*}\right) / \kappa_{v}\left(\mathcal{P}_{n}^{*}\right)$. Thus, by definition of $\kappa_{v}$,

$$
\begin{gathered}
E\left[f\left(\frac{\lambda_{1, h}\left(T_{n, h}\right)}{\lambda_{1, h}\left(T_{n, h}-\right)}, \frac{\lambda_{2, h}\left(T_{n, h}\right)}{\lambda_{1, h}\left(T_{n, h}-\right)}, \ldots, \frac{\lambda_{l, h}\left(T_{n, h}\right)}{\lambda_{1, h}\left(T_{n, h}-\right)}\right) 1_{\left\{A_{\left(n_{1}, n_{2}, \ldots, n_{l}\right), h}\right\}}\right] \\
=\frac{1}{\kappa_{v}\left(\mathcal{P}_{n}^{*}\right)} \int_{\mathcal{S} \downarrow} E\left[f\left(\Pi_{s, 1}, \ldots, \Pi_{s, l}\right) 1_{\left\{A_{\left(n_{1}, n_{2}, \ldots, n_{l}\right), h}^{s}\right]}\right] v(d s),
\end{gathered}
$$

where $A_{\left(n_{1}, n_{2}, \ldots, n_{l}\right), h}^{s}$ is defined as $A_{\left(n_{1}, n_{2}, \ldots, n_{l}\right), h}$ by replacing in (A.2) $\Delta\left(T_{n, h}\right)$ by $\Pi_{s}$. It is then easy to check with the definition of $\Pi_{s}$ that the required formula holds.

Proof of Lemma 2. The first part of the proof consists in shifting the problem to a homogeneous fragmentation with the same dislocation measure $v$. This can be done by using the construction of self-similar fragmentations from homogeneous ones recalled in Section 2. So, consider a homogeneous interval fragmentation $F_{h}$ from which we construct the $\alpha$-self-similar one by time-change (1). In this homogeneous fragmentation, tag independently $n$ fragments as in the previous lemma. Keeping the notation introduced there, is easy to see that

$$
\left(\lambda_{1, h}\left(T_{n, h}\right), \ldots, \lambda_{n, h}\left(T_{n, h}\right), 1_{\left\{A_{\left(n_{1}, n_{2}, \ldots, n_{l}, h\right.}\right\}}\right) \stackrel{\text { law }}{=}\left(\lambda_{1}\left(T_{n}\right), \ldots, \lambda_{n}\left(T_{n}\right) 1_{\left\{A_{\left(n_{1}, n_{2}, \ldots, n_{l}\right)}\right\}}\right) .
$$

So that the aim of this proof is to find for which $l$-tuples $\left(a_{1}, \ldots, a_{l}\right)$, the expectation

$$
E\left[\prod_{k=1}^{l} \lambda_{k, h}^{-a_{k}}\left(T_{n, h}\right) 1_{\left\{\lambda_{1, h}\left(T_{n, h}\right) \geqslant \lambda_{2, h}\left(T_{n, h}\right) \geqslant \cdots \geqslant \lambda_{l, h}\left(T_{n, h}\right)\right\}} 1_{\left\{A_{\left(n_{1}, n_{2}, \ldots, n_{l}\right), h}\right\}}\right]
$$

is finite.

By Lemma 14, we have that

$$
\begin{aligned}
& E\left[\prod_{k=1}^{l} \lambda_{k, h}^{-a_{k}}\left(T_{n, h}\right) 1_{\left\{\lambda_{1, h}\left(T_{n, h}\right) \geqslant \cdots \geqslant \lambda_{l, h}\left(T_{n, h}\right)\right\}} 1_{\left\{A_{\left(n_{1}, n_{2}, \ldots, n, h\right.}\right\}}\right] \\
& \quad=E\left[\left(\lambda_{1, h}\left(T_{n, h}-\right)\right)^{-\sum_{k=1}^{l} a_{k}}\right] E\left[\prod_{k=1}^{l}\left(\frac{\lambda_{k, h}\left(T_{n, h}\right)}{\lambda_{1, h}\left(T_{n, h}-\right)}\right)^{-a_{k}} 1_{\left\{\lambda_{1, h}\left(T_{n, h}\right) \geqslant \cdots \geqslant \lambda_{l, h}\left(T_{n, h}\right)\right\}} 1_{\left\{A_{\left(n_{1}, n_{2}, \ldots, n_{l}\right), h}\right\}}\right]
\end{aligned}
$$


and that

$$
\begin{aligned}
& E\left[\prod_{k=1}^{l}\left(\frac{\lambda_{k, h}\left(T_{n, h}\right)}{\lambda_{1, h}\left(T_{n, h}-\right)}\right)^{-a_{k}} 1_{\left\{\lambda_{1, h}\left(T_{n, h}\right) \geqslant \cdots \geqslant \lambda_{l, h}\left(T_{n, h}\right)\right\}} 1_{\left\{A_{n_{1}, n_{2}, \ldots, n_{l}, h}\right\}}\right]<\infty \\
& \quad \Leftrightarrow \int_{\mathcal{S} \downarrow} \sum_{i_{1}<\cdots<i_{l}} \prod_{k=1}^{l} s_{i_{k}}^{n_{k}-a_{k}} 1_{\left\{s_{i_{k}}>0\right\}} v(d s)<\infty .
\end{aligned}
$$

So it just remains to specify for which $\left(a_{1}, \ldots, a_{l}\right)$, the expectation $E\left[\left(\lambda_{1, h}\left(T_{n, h}-\right)\right)^{-\sum_{k=1}^{l} a_{k}}\right]$ is finite. To that end, remark that given $\lambda_{1, h}$, the probability that the tagged points $U_{2, h}, \ldots, U_{n, h}$ belong to the same fragment as $U_{1, h}$ at time $t$ is equal to $\lambda_{1, h}^{n-1}(t)$, since the $U_{i, h}$ 's are independent and uniformly distributed on ]0, 1[. In other words,

$$
P\left(T_{n, h}>t \mid \lambda_{1, h}\right)=\lambda_{1, h}^{n-1}(t) \quad \forall t>0 .
$$

As recalled in Section 3, the process $\left(\lambda_{1, h}(t), t \geqslant 0\right)$ can be expressed in the form (exp $\left.\left(-\xi_{t}\right), t \geqslant 0\right)$, for some pure jumps subordinator $\xi$ with Laplace exponent $\phi$ given by (4). Therefore $P\left(T_{n, h}>t \mid \lambda_{1, h}\right)=\mathrm{e}^{-(n-1) \xi_{t}}$ and for all $a \in \mathbb{R}:$

$$
\begin{aligned}
E\left[\lambda_{1, h}^{-a}\left(T_{n, h}-\right)\right] & =E\left[\int_{0}^{\infty} \mathrm{e}^{a \xi_{t^{-}}} P\left(T_{n, h} \in d t \mid \lambda_{1, h}\right)\right] \\
& =E\left[\int_{0}^{\infty} \sum_{0<s<t}\left(\mathrm{e}^{a \xi_{s}}-\mathrm{e}^{a \xi_{s^{-}}}\right) P\left(T_{n, h} \in d t \mid \lambda_{1, h}\right)\right]+1 \\
& =E\left[\sum_{0<s<\infty}\left(\mathrm{e}^{a \xi_{s}}-\mathrm{e}^{a \xi_{s^{-}}}\right) \mathrm{e}^{-(n-1) \xi_{s}}\right]+1 \\
& =E\left[\sum_{0<s<\infty} \mathrm{e}^{(a-(n-1)) \xi_{s^{-}}}\left(\mathrm{e}^{(a-(n-1)) \Delta_{s}}-\mathrm{e}^{-(n-1) \Delta_{s}}\right)\right]+1 \quad\left(\Delta_{s}=\xi_{s}-\xi_{s^{-}}\right) .
\end{aligned}
$$

Finally, using the Master Formula (see [22], p. 475), we get

$$
E\left[\lambda_{1, h}^{-a}\left(T_{n, h}-\right)\right]=E\left[\int_{0}^{\infty} \mathrm{e}^{(a-(n-1)) \xi_{s}} d s\right] \int_{0}^{\infty}\left(\mathrm{e}^{(a-(n-1)) x}-\mathrm{e}^{-(n-1) x}\right) \pi(d x)+1,
$$

$\pi$ being the Lévy measure of $\xi$. The integral $\int_{0}^{\infty}\left(\mathrm{e}^{(a-(n-1)) x}-\mathrm{e}^{-(n-1) x}\right) \pi(d x)$ is finite as soon as $a \leqslant n-1$ and the expectation $E\left[\int_{0}^{\infty} \mathrm{e}^{(a-(n-1)) \xi_{s}} d s\right]$ is finite if and only if $a<n-1$, since $E\left[\mathrm{e}^{-q \xi_{s}}\right]=\mathrm{e}^{-s \phi(q)}$ where $\phi>0$ on ] $0, \infty[, \phi \in[-\infty, 0]$ on $]-\infty, 0]$. This completes the proof.

\section{References}

[1] D.J. Aldous, Deterministic and stochastic models for coalescence (aggregation and coagulation): a review of the mean-field theory for probabilists, Bernoulli 5 (1999) 3-48.

[2] E. Artin, The Gamma Function, Holt, Rinehart, and Winston, New York, 1964.

[3] J. Berestycki, Ranked fragmentations, ESAIM P\&S 6 (2002) 157-176.

[4] J. Bertoin, Subordinators: Examples and applications, in: P. Bernard (Ed.), Lectures on Probability Theory and Statistics, Ecole d'été de probabilités de St-Flour XXVII, in: Lect. Notes in Maths., vol. 1717, Springer, Berlin, 1999, pp. 1-91.

[5] J. Bertoin, Homogeneous fragmentation processes, Probab. Theory Related Fields 121 (3) (2001) 301-318.

[6] J. Bertoin, Self-similar fragmentations, Ann. Inst. Henri Poincaré 38 (2002) 319-340. 
[7] J. Bertoin, The asymptotic behavior of fragmentation processes, J. Eur. Math. Soc. 5 (2003) 395-416.

[8] J. Bertoin, On small masses in self-similar fragmentations, Stochastic Process. Applic. 109 (2004) 13-22.

[9] J. Bertoin, M. Yor, On subordinators, self-similar Markov processes and factorization of the exponential variable, Elect. Comm. Probab. 6 (10) (2001) 95-106.

[10] D. Beysens, X. Campi, E. Pefferkorn (Eds.), Proceedings of the Workshop: Fragmentation Phenomena, Les Houches Series, World Scientific, 1995.

[11] N.H. Bingham, C.M. Goldie, J.L. Teugels, Regular Variation, Cambridge University Press, 1987.

[12] S. Bochner, K. Chandrasekharan, Fourier Transforms, Princeton University Press, 1949.

[13] P. Carmona, F. Petit, M. Yor, On the distribution and asymptotic results for exponential functionals of Lévy processes, in: M. Yor (Ed.), Exponential Functionals and Principal Values Related to Brownian Motion, Biblioteca de la Revista Matematica IberoAmericana, 1997, pp. 73-121.

[14] K. Falconer, The Geometry of Fractal Sets, Cambridge University Press, 1986.

[15] A.F. Filippov, On the distribution of the sizes of particles which undergo splitting, Theory Probab. Appl. 6 (1961) $275-294$.

[16] N. Fournier, J.S. Giet, On small particles in coagulation-fragmentation equations, J. Statist. Phys. 111 (5) (2003) 1299-1329.

[17] B. Haas, Loss of mass in deterministic and random fragmentations, Stochastic Process. Appl. 106 (2) (2003) 245-277.

[18] B. Haas, G. Miermont, The genealogy of self-similar fragmentations with negative index as a continuum random tree, Electron. J. Probab., submitted for publication.

[19] I. Jeon, Stochastic fragmentation and some sufficient conditions for shattering transitions, J. Korean Math. Soc. 39 (4) (2002) $543-558$.

[20] J.F.C. Kingman, The coalescent, Stochastic Process. Appl. 13 (1982) 235-248.

[21] G. Miermont, Self-similar fragmentations derived from the stable tree I: splitting at heights, Probab. Theory Related Fields 127 (2003) 423-454.

[22] D. Revuz, M. Yor, Continuous Martingales and Brownian Motion, third ed., Springer, 1998.

[23] K.-I. Sato, Lévy Processes and Infinitely Divisible Distributions, Cambridge University Press, 1999.

[24] E.M. Stein, Singular Integrals and Differentiability Properties of Functionals, Princeton University Press, 1970. 